\title{
Recent progress in random metric theory and its applications to conditional risk measures
}

\author{
Tiexin Guo* \\ LMIB and School of Mathematics and Systems Science, Beihang University, \\ Beijing 100191, P.R. China.
}

\begin{abstract}
The purpose of this paper is to give a selective survey on recent progress in random metric theory and its applications to conditional risk measures. This paper includes eight sections. Section 1 is a longer introduction, which gives a brief introduction to random metric theory, risk measures and conditional risk measures. Section 2 gives the central framework in random metric theory, topological structures, important examples, the notions of a random conjugate space and the Hahn-Banach theorems for random linear functionals. Section 3 gives several important representation theorems for random conjugate spaces. Section 4 gives characterizations for a complete random normed module to be random reflexive. Section 5 gives hyperplane separation theorems currently available in random locally convex modules. Section 6 gives the theory of random duality with respect to the locally $L^{0}$-convex topology and in particular a characterization for a locally $L^{0}$-convex module to be $L^{0}$-pre-barreled. Section 7 gives some basic results on $L^{0}$-convex analysis together with some applications to conditional risk measures. Finally, Section 8 is devoted to extensions of conditional convex risk measures, which shows that every representable $L^{\infty}-$ type of conditional convex risk measure and every continuous $L^{p}$-type of convex conditional risk measure $(1 \leqslant p<$ $+\infty)$ can be extended to an $L_{\mathcal{F}}^{\infty}(\mathcal{E})$-type of $\sigma_{\epsilon, \lambda}\left(L_{\mathcal{F}}^{\infty}(\mathcal{E}), L_{\mathcal{F}}^{1}(\mathcal{E})\right)$-lower semicontinuous conditional convex risk measure and an $L_{\mathcal{F}}^{p}(\mathcal{E})$-type of $\mathcal{T}_{\epsilon, \lambda}$-continuous conditional convex risk measure $(1 \leqslant p<+\infty)$, respectively.
\end{abstract}

Keywords: random normed module, random inner product module, random locally convex module, random conjugate space, $L^{0}$-convex analysis, conditional risk measures

MSC(2000): 46A22, 46A25, 46H25, 47H40, 52A41, 91B16, 91B30, 91B70.

\section{Introduction}

In the last ten years random metric theory and its applications have undergone a systematic and deep development, in particular random metric theory recently has been a proper mathematical tool for the study of conditional risk measures for unbounded financial positions. The purpose of this section is to give a brief historic retrospect to the respective courses of the development of random metric theory, risk measures and conditional risk measures in order to make it easier for the reader to see how random metric theory and the theory of conditional risk measures come together.

\subsection{The central framework in random metric theory}

Random metric theory originated from the theory of probabilistic metric spaces $[33,34,39,63]$. The central framework in random metric theory was formed in the course of the development of random

* E-mail address: txguo@buaa.edu.cn

Supported by NNSF No. 10871016 
metric theory in the direction of functional analysis[24-28, 30, 31, 46, 47, 68]. A crucial step in the formative process was taken in [31], where the respective new versions of the notions of a random metric space and random normed space originally introduced in [63] were presented. According to the new versions the random norm of a vector in a random normed space (resp., the random distance between any two points in a random metric space) is the equivalence class of a nonnegative random variable rather than a nonnegative random variable as defined in [63]. Since a random normed space is often endowed with a natural topology, called the $(\epsilon, \lambda)$-topology, it is not a locally convex linear topological space in general and the theory of traditional conjugate spaces universally fails to serve the study of random normed spaces, for example, our recent result in [48] shows that for a special class of random normed spaces - random normed modules with base $(\Omega, \mathcal{F}, P)$ (a probability space) they admit sufficiently many nontrivial continuous linear functionals iff $\mathcal{F}$ is generated by at most countably many $P$-atoms, so the development of random normed spaces needs a new kind of conjugate space theory. Motivated by the study of random linear operators in random functional analysis and based on the earlier work in [24-27], Guo presented in [31] the definitive notion of an almost surely bounded random linear functional and proved the Hahn-Banach theorem for such random linear functionals, which led us directly to the idea of random conjugate spaces. However, the structure of random normed spaces is too weak to guarantee that an almost surely bounded random linear functional defined on a random normed space possesses nice properties, so that the deep development of the theory of random conjugate spaces encounters a serious obstacle. We found in [26] that in order to ensure an almost surely bounded random linear functional on a random normed space to possess pleasant properties the random normed space has to possess a kind of module structure, which motivated us to present the notion of a random normed module in [26]. Based on the new version given in [31] of a random normed space, Guo further presented in [31] the elaborated versions of the notions of a random normed module and random inner product module originally introduced in $[26,46]$ respectively, which also leads to the definitive notion of the random conjugate space of a random normed space, namely, the random conjugate space of a random normed space is exactly the random normed module consisting of all almost surely bounded random linear functionals defined on the random normed space. The results in [26] show that only the theory of random conjugate spaces for random normed modules can be deeply developed, consequently, the center of our work has been placed at the topics closely related to the theory of random conjugate spaces of random normed modules since 1995. Subsequently, we established the representation theorems of random conjugate spaces in $[27,46]$ and studied module homomorphisms on random normed modules in [28]. Motivated by the work on the representation of the dual of Lebesgue-Bochner function spaces [12], we established in [30,32] the precise connection between the random conjugate space $S^{*}$ of a random normed module $S$ and the classical conjugate space $\left(L^{p}(S)\right)^{\prime}$ of the abstract normed space $L^{p}(S)$ generated from $S$, namely

$$
\left(L^{p}(S)\right)^{\prime} \cong L^{q}\left(S^{*}\right) \quad\left(1 \leqslant p<+\infty \quad \text { and } \quad \frac{1}{p}+\frac{1}{q}=1\right) .
$$

Making use of this connection, we established various characterizations for a complete random normed module to be random reflexive [30,42], and a basic strict separation theorem in random locally convex modules [45]. The notion of a random locally convex module was introduced in [34] in order to provide a proper framework for the further development of the theory of random conjugate 
spaces of random normed modules, subsequently random $\mathrm{w}^{*}$-topology and random weak topology were thoroughly studied in [37], and random duality was also developed in [41].

Now, random normed modules, random inner product modules and random locally convex modules have become the central framework supporting random metric theory, and the theory of random conjugate spaces has been a powerful tool for the development of the central framework. We can now say that random metric theory is being developed as functional analysis founded on the central framework. In the course of development, the theory of random normed modules together with their random conjugate spaces has found many successful applications in solving the best approximation problem and the dual representation problem in Lebesgue-Bochner function spaces [32, 47, 68], in geometry of Banach spaces [27, 49], and in solving various measurability problems [36, 40].

\subsection{Risk measures and classical convex analysis}

Risk measures were introduced in order to quantify the riskiness of financial positions and to provide a criterion to determine whether the risk was acceptable or not. Since Artzner et al. and Delbaen presented and studied coherent risk measures in their seminal papers $[1,10]$ for the model space $L^{\infty}$ (namely the Banach space of essentially bounded random variables, which is used to model the essentially bounded financial positions), the theory of risk measures has obtained a quite extensive development. In 2002, convex risk measures broader than coherent risk measures were presented and studied by Föllmer and Schied in [19-21] and also independently by Frittelli and Rosazza Gianin in $[22,23]$. Since the model space is too narrow to include the important risk models such as normally or log-normally distributed random variables, there is a growing mathematical finance literature dealing with convex risk measures beyond $L^{\infty}$, see e.g., [3, 8, 9, 17, 53, 55, 61]. Since convex risk measures are extended real-valued convex functions defined on locally convex spaces such as $L^{p}$ $(1 \leqslant p \leqslant+\infty)$, classical convex analysis[14,59] turns out to be a powerful tool for the analysis of convex risk measures, cf. [23].

\subsection{Conditional risk measures and random metric theory}

Conditional risk measures were introduced in order to quantify the risk associated with financial positions when the additional information was available. Various interpretations of the additional information in $[4,11]$ show that the theory of conditional risk measures open a way to the analysis of the consequences of asymmetric information for risk measurement.

To briefly introduce them, let $(\Omega, \mathcal{E}, P)$ be a probability space, $L^{p}(\mathcal{E})$ the Banach spaces of equivalence classes of real-valued $p$-integrable or essentially bounded (according to $1 \leqslant p<+\infty$ or $p=+\infty) \mathcal{E}$-measurable random variables on $\Omega, \bar{L}^{0}(\mathcal{E})$ (or, $L^{0}(\mathcal{E})$ ) the set of equivalence classes of $\mathcal{E}$-measurable extended real-valued (real-valued) random variables on $\Omega$, and $\mathcal{F}$ a sub $\sigma$-algebra of $\mathcal{E}$, which denotes the additional information.

The first definition of a conditional risk measure, here we call it a conditional risk measure of $L^{\infty}$-type, was introduced by Detlefsen and Scandolo in [11] and independently by Bion-Nadal in [4] as follows:

Definition 1.3.1 $[4,11] . \quad$ A function $f: L^{\infty}(\mathcal{E}) \rightarrow L^{\infty}(\mathcal{F})$ is called: 
(1) $L^{0}(\mathcal{F})$-convex if $f(\xi x+(1-\xi) y) \leqslant \xi f(x)+(1-\xi) f(y), \forall x, y \in L^{\infty}(\mathcal{E})$ and $\xi \in L_{+}^{0}(\mathcal{F})$ such that $0 \leqslant \xi \leqslant 1$;

(2) monotone if $f(x) \leqslant f(y)$ for all $x$ and $y$ in $L^{\infty}(\mathcal{E})$ such that $x \geqslant y$;

(3) cash invariant if $f(x+y)=f(x)-y$ for all $x$ in $L^{\infty}(\mathcal{E})$ and $y$ in $L^{\infty}(\mathcal{F})$.

Furthermore, $f$ is called a conditional convex risk measure of $L^{\infty}$-type if it is $L^{0}(\mathcal{F})$-convex, monotone and cash invariant.

Let $\mathcal{P}$ be the set of all the probability measures $Q$ on $\mathcal{E}$ such that $Q$ is absolutely continuous with respect to $P$ and $\mathcal{P}_{\mathcal{F}}=\{Q \in \mathcal{P} \mid Q=P$ on $\mathcal{F}\}$

Given a conditional convex risk measure $f, \alpha: \mathcal{P}_{\mathcal{F}} \rightarrow \bar{L}^{0}(\mathcal{F})$ is defined by $\alpha(Q)=\vee\left\{E_{Q}(-x \mid \mathcal{F})-\right.$ $\left.f(x) \mid x \in L^{\infty}(\mathcal{E})\right\}$ for any $Q$ in $\mathcal{P}_{\mathcal{F}}$, called the random penalty function of $f$, where $E_{Q}(\cdot \mid \mathcal{F})$ denotes the conditional expectation given the $\sigma$-algebra $\mathcal{F}$ under the probability $Q$. The following dual representation proposition was proved by Detlefsen and Scandolo in [11], see [4] for dual representation under a stronger assumption that $f$ is continuous from below and [18] for other possible forms of dual representation.

Proposition 1.3.1 [11]. The following three statements are equivalent to each other:

(1) $f(x)=\vee\left\{E_{Q}(-x \mid \mathcal{F})-\alpha(Q) \mid Q \in \mathcal{P}_{\mathcal{F}}\right\}, \forall x \in L^{\infty}(\mathcal{E})$;

(2) $f$ is continuous from above, namely $f\left(x_{n}\right) \nearrow f(x)$ whenever $x_{n} \searrow x$;

(3) $f$ has the "Fatou property": For any bounded sequence $\left\{x_{n}, n \in N\right\}$ which converges P-a.s. to some $x, f(x) \leqslant \liminf _{n \uparrow \infty} f\left(x_{n}\right)$.

From the essence of the proof of Proposition 1.3.1 given in [11], one can see that classical convex analysis may still treat the conditional convex risk measure of $L^{\infty}$-type. Based on this kind of conditional convex risk measure, the corresponding dynamic risk measures of $L^{\infty}$-type were developed in $[11,18]$, see $[6,7]$ for $L^{\infty}$-type of dynamic risk measures of bounded stochastic processes.

Just as stated above in Section 1.2, $L^{\infty}(\mathcal{E})$ as the model space for conditional risk measures is too narrow. Recently, motivated by the study of dynamic risk measures $[58,60]$ and conditional entropic risk measures, Filipović, Kupper and Vogelpoth studied the following convex conditional risk measures of $L_{\mathcal{F}}^{p}(\mathcal{E})$-type and $L^{p}$-type in $[15,16,56]$.

Let us first introduce a conditional risk measure of $L^{p}$-type.

Definition 1.3.2 [16]. Let $1 \leqslant r \leqslant p<\infty$, then a function $f: L^{p}(\mathcal{E}) \rightarrow L^{r}(\mathcal{F})$ is called

(1) $L^{0}(\mathcal{F})$-convex if $f(\xi x+(1-\xi) y) \leqslant \xi f(x)+(1-\xi) f(y), \forall x, y \in L^{p}(\mathcal{E})$ and $\xi \in L_{+}^{0}(\mathcal{F})$ such that $0 \leqslant \xi \leqslant 1$;

(2) monotone if $f(x) \leqslant f(y)$ for all $x$ and $y$ in $L^{p}(\mathcal{E})$ such that $x \geqslant y$;

(3) cash invariant if $f(x+y)=f(x)-y$ for all $x$ in $L^{p}(\mathcal{E})$ and $y$ in $L^{p}(\mathcal{F})$;

(4) local if $\tilde{I}_{A} f(x)=\tilde{I}_{A} f\left(\tilde{I}_{A} x\right)$ for all $A \in \mathcal{F}$ and $x \in L^{p}(\mathcal{E})$.

Furthermore, $f$ is called a convex conditional risk measure of $L^{p}$-type if it is convex (namely convex in the usual sense), monotone and cash invariant.

Given a function $f: L^{p}(\mathcal{E}) \rightarrow L^{r}(\mathcal{F})$, the function $f^{*}: B\left(L^{p}(\mathcal{E}), L^{r}(\mathcal{F})\right) \rightarrow \bar{L}^{0}(\mathcal{F})$ is defined by $f^{*}(u)=\vee\left\{u(x)-f(x) \mid x \in L^{p}(\mathcal{E})\right\}$ for all $u \in B\left(L^{p}(\mathcal{E}), L^{r}(\mathcal{F})\right)$, where $B\left(L^{p}(\mathcal{E}), L^{r}(\mathcal{F})\right)$ denotes the Banach space of continuous linear operators from $L^{p}(\mathcal{E})$ to $L^{r}(\mathcal{F})$, further let $\operatorname{dom}\left(f^{*}\right)=\{u \in$ $\left.B\left(L^{p}(\mathcal{E}), L^{r}(\mathcal{F})\right) \mid f^{*}(u) \in L^{r}(\mathcal{F})\right\}$. Finally, $u \in B\left(L^{p}(\mathcal{E}), L^{r}(\mathcal{F})\right)$ is called a subgradient of $f$ at 
$x_{0} \in L^{p}(\mathcal{E})$ if $u\left(x-x_{0}\right) \leqslant f(x)-f\left(x_{0}\right), \forall x \in L^{p}(\mathcal{E})$, the set of subgradients of $f$ at $x_{0}$ is denoted by $\partial f\left(x_{0}\right)$.

Zowe proved the following in [69]:

Proposition 1.3.2. Let $f$ be a convex function from $L^{p}(\mathcal{E})$ to $L^{r}(\mathcal{F})$ and continuous at $x_{0}$. Then $\partial f\left(x_{0}\right) \neq \emptyset$ and $f\left(x_{0}\right)=\vee\left\{u\left(x_{0}\right)-f^{*}(u) \mid u \in \operatorname{dom}\left(f^{*}\right)\right\}$.

In fact, we can prove that a convex conditional risk measure of $L^{p}$-type is $L^{0}(\mathcal{F})$-convex if it is continuous. Recently, Filipović et al. proved the following in [16]:

Proposition 1.3.3. Let $f$ be a continuous convex conditional risk measure from $L^{p}(\mathcal{E})$ to $L^{r}(\mathcal{F})$. Then $\partial f(x) \neq \emptyset$ for all $x$ in $L^{p}(\mathcal{E})$ and $f(x)=\vee\left\{E(x y \mid \mathcal{F})-f^{*}(E(\cdot y \mid \mathcal{F})) \mid y \in L^{q}(\mathcal{E}), y \leqslant\right.$ $0, E(y \mid \mathcal{F})=-1$ and $\left.E\left(|y|^{q} \mid \mathcal{F}\right) \in L^{\frac{r(p-1)}{p-r}}(\mathcal{F})\right\}$, where $q$ is the Hölder conjugate number of $p$, $\frac{r(p-1)}{p-r}=\infty$ when $p=r$ and $E(\cdot y \mid \mathcal{F}): L^{p}(\mathcal{E}) \rightarrow L^{r}(\mathcal{F})$ is defined by $E(\cdot y \mid \mathcal{F})(x)=E(x y \mid \mathcal{F}), \forall x \in$ $L^{p}(\mathcal{E})$.

From the essence of the proofs of Propositions 1.3.2 and 1.3.3 given in [16], the vector-valued convex analysis and a bit of basic linear operator theory can still treat a convex conditional risk measure of $L^{p}$-type.

Let $1 \leqslant p \leqslant+\infty$ and $L_{\mathcal{F}}^{p}(\mathcal{E})=$ the $L^{0}(\mathcal{F})$-module generated by $L^{p}(\mathcal{E})$, namely $L_{\mathcal{F}}^{p}(\mathcal{E})=L^{0}(\mathcal{F})$. $L^{p}(\mathcal{E}):=\left\{\xi x: \xi \in L^{0}(\mathcal{F})\right.$ and $\left.x \in L^{p}(\mathcal{E})\right\}$, which can be made a random normed module in a natural way. Then the following conditional risk measure of $L_{\mathcal{F}}^{p}(\mathcal{E})$-type was studied in $[15,56]$ and eventually presented in [16]:

Definition 1.3.3 [16]. Let $1 \leqslant p \leqslant+\infty$. A function $f: L_{\mathcal{F}}^{p}(\mathcal{E}) \rightarrow \bar{L}^{0}(\mathcal{F})$ is called:

(1) monotone if $f(x) \leqslant f(y)$ for all $x, y \in L_{\mathcal{F}}^{p}(\mathcal{E})$ such that $x \geqslant y$;

(2) subcash invariant if $f(x+y) \geqslant f(x)-y$ for all $x \in L_{\mathcal{F}}^{p}(\mathcal{E})$ and $y \in L_{+}^{0}(\mathcal{F})$;

(3) cash invariant if $f(x+y)=f(x)-y$ for all $x \in L_{\mathcal{F}}^{p}(\mathcal{E})$ and $y \in L^{0}(\mathcal{F})$;

Further, an $L^{0}(\mathcal{F})$-convex, monotone and cash invariant function from $L_{\mathcal{F}}^{p}(\mathcal{E})$ to $\bar{L}^{0}(\mathcal{F})$ is called a conditional convex risk measure of $L_{\mathcal{F}}^{p}(\mathcal{E})$-type.

A typical example motivating Filipović, Kupper and Vogelpoth to present and study a conditional convex risk measure of $L_{\mathcal{F}}^{p}(\mathcal{E})$-type is the following conditional entropic risk measure $\rho_{\gamma}(\cdot): \bar{L}^{0}(\mathcal{E}) \rightarrow$ $\bar{L}^{0}(\mathcal{F})$ defined by $\rho_{\gamma}(x)=\frac{1}{\gamma} \log E\left(e^{-\gamma x} \mid \mathcal{F}\right), \forall x \in \bar{L}^{0}(\mathcal{E})$, where $\gamma>0$ is a risk aversion.

When $\rho_{\gamma}$ is restricted to $L^{\infty}(\mathcal{E}), \rho_{\gamma}$ is a conditional convex risk measure of $L^{\infty}$-type continuous from above, then the dual representation of $\rho_{\gamma}$ can be treated as in $[4,11]$.

When $\rho_{\gamma}$ is restricted to $L^{p}(\mathcal{E})(1 \leqslant p<+\infty), \rho_{\gamma}$ is an $L^{0}(\mathcal{F})$-convex proper lower semicontinuous function from $L^{p}(\mathcal{E})$ to $\bar{L}^{0}(\mathcal{F})$, the methods used to establish the dual representation results of conditional convex risk measures of $L^{\infty}$-type and $L^{p}$-type no longer apply to $\rho_{\gamma}$.

Motivated by the idea of hedging random future payments in a multiperiod setting and also led by giving a more pleasant dual representation result for $\rho_{\gamma}$ than that as given in $[4,11]$, Filipović et al. presented in [15] the idea of randomizing the initial data, for example, randomizing the initial data $L^{p}(\mathcal{E})$ into the random normed module $L_{\mathcal{F}}^{p}(\mathcal{E})$. Further, $\rho_{\gamma}$ is regarded as a mapping from $L_{\mathcal{F}}^{p}(\mathcal{E})$ to $\bar{L}^{0}(\mathcal{F})$, then it is a conditional convex risk measure of $L_{\mathcal{F}}^{p}(\mathcal{E})$-type and is also lower semicontinuous in the sense of [15]. To establish the dual representation result for this kind of conditional convex risk measures such as $\rho_{\gamma}$ on $L_{\mathcal{F}}^{p}(\mathcal{E})$, Filipović, Kupper and Vogelpoth attempted to carry out a spectacular generalization of the classical Fenchel-Moreau type dual representation 
theorem from locally convex spaces to random locally convex modules by substituting random locally convex modules for classical locally convex spaces and random conjugate spaces for classical conjugate spaces. Further, to establish the continuity and subdifferentiability theorems for proper lower semicontinuous $L^{0}$-convex functions on random locally convex modules, they also introduced in [15] the locally $L^{0}$-convex topology much stronger than the $(\epsilon, \lambda)$-topology for random locally convex modules. Besides, they also established in [15] two Hyperplane separation theorems in order to establish the subdifferentiability and Fenchel-Moreau type dual representation theorems for $L^{0}$ convex functions on locally $L^{0}$-convex modules (a locally $L^{0}$-convex module amounts to a random locally convex module endowed with the locally $L^{0}$-convex topology).

In [39], Guo simultaneously considered the two kinds of topologies - the $(\epsilon, \lambda)$-topology and locally $L^{0}$-convex topology for a random locally convex module in order to give the relations between the basic results currently available derived from the two kinds of topologies. Consequently, it was proved in [39] that our basic strict separation theorem earlier established in [35, 44, 45] implies the hyperplane separation theorem in [15] which was used to establish the generalized Fenchel-Moreau type dual representation theorem in [15], that the two kinds of random conjugate spaces derived from the two kinds of topologies coincide for most of random locally convex modules, and in particular that a random locally convex module has the same completeness under the two kinds of topologies if the module has the countable concatenation property as defined in [39]. The results in [39] further show that most of the previously established principal results of random conjugate spaces of random normed modules under the $(\epsilon, \lambda)$-topology are still valid under the locally $L^{0}$-convex topology, which considerably enriches financial applications of random normed modules.

It was already pointed out in [39] that the new countable concatenation property introduced in [39] will play an essential role in the study of locally $L^{0}$-convex modules. Our recent work[50] has improved the results obtained in [15] for separation and duality in locally $L^{0}$-convex modules. Further, we developed the theory of random duality with respect to the locally $L^{0}$-convex topology [50], which leads us directly to the notion of an $L^{0}$-pre-barreled module that is weaker than the notion of an $L^{0}$-barreled module originally introduced in [15]. What is important is that under the weaker definition we can establish the characterization for a locally $L^{0}$-convex module to be $L^{0}$-pre-barreled, which shows that a complete random normed module with the countable concatenation property is $L^{0}$-pre-barreled under the locally $L^{0}$-convex topology, in particular, $L_{\mathcal{F}}^{p}(\mathcal{E})$ is $L^{0}$-pre-barreled. Therefore this weaker notion is more suitable for the study of the continuity and subdifferentiability of a proper lower semicontinuous conditional convex risk measure of $L_{\mathcal{F}}^{p}(\mathcal{E})$-type. In particular, we can also establish the $(\epsilon, \lambda)$-topological version of Fenchel-Moreau dual representation theorem for proper lower semicontinuous $L^{0}$-convex functions [50], which not only contains the locally $L^{0}$ convex topological version of Fenchel-Moreau dual representation theorem established in [15] as a special case but also seems more natural than the latter. The final part of this paper shows that a conditional convex risk measure of either $L^{\infty}$-type or $L^{p}$-type can always be extended to one of $L_{\mathcal{F}}^{p}(\mathcal{E})$-type and that the representation propositions obtained under Definition 1.3.3 have included Propositions 1.3 .1 and 1.3.3 as special cases, which together with the work[15, 16, 39, 50, 56] has paved the way for unifiedly developing the theory of conditional risk measures, so that the theory of random locally convex modules, in particular random normed modules together with their random conjugate spaces is a mathematical tool tailored to the theory of conditional risk measures. We 
believe that the further development of dynamic risk measures will involve more of random metric theory.

The purpose of this paper is to give a selective survey on the recent progress in random metric theory and its applications to conditional risk measures.

This paper includes eight sections. The first is the very introduction, and the remainder of this paper is organized as follows. Section 2 gives the central framework in random metric theory, topological structures, important examples, the notions of a random conjugate space and the HahnBanach theorems for random linear functionals; Section 3 gives several important representation theorems for random conjugate spaces; Section 4 gives characterizations for a complete random normed module to be random reflexive; Section 5 gives hyperplane separation theorems currently available in random locally convex modules; Section 6 gives the theory of random duality with respect to the locally $L^{0}$-convex topology and in particular a characterization for a locally $L^{0}$-convex module to be $L^{0}$-pre-barreled; Section 7 gives some basic results on $L^{0}$-convex analysis together with some applications to conditional risk measures; Finally, Section 8 is devoted to extensions of conditional risk measures.

Throughout this paper, $(\Omega, \mathcal{F}, P)$ denotes a given probability space, $K$ the scalar field $R$ of real numbers or $C$ of complex numbers and $L^{0}(\mathcal{F}, K)$ the algebra over $K$ of equivalence classes of $K$ valued $\mathcal{F}$-measurable random variables on $\Omega$.

Proposition 1.3.4 below is the well known theorem on the existence of an essential supremum for a set of random variables. Let $\overline{\mathcal{L}}^{0}(\mathcal{F}, R)$ be the set of extended real-valued $\mathcal{F}$-measurable random variables on $(\Omega, \mathcal{F}, P)$ and $H$ a subset of $\overline{\mathcal{L}}^{0}(\mathcal{F}, R) . \xi \in \overline{\mathcal{L}}^{0}(\mathcal{F}, R)$ is called an essential upper bound for $H$ if $\eta(\omega) \leqslant \xi(\omega) P-a . s$. (namely $P$-almost surely) for each $\eta \in H$; furthermore if for each essential upper bound $\eta^{\prime}$ for $H$ it always holds that $\eta(\omega) \leqslant \eta^{\prime}(\omega) P$-a.s., then the essential upper bound $\eta$ is called an essential supremum for $H$, denoted by $\operatorname{esssup}(H)$. Obviously, $\operatorname{esssup}(H)$ is unique $P-$ a.s. Similarly, one has the notion of an essential infimum.

Proposition 1.3.4 [52]. Every subset $H$ of $\overline{\mathcal{L}}^{0}(\mathcal{F}, R)$ has an essential supremum and an essential infimum, and there exist countable subsets $\left\{a_{n}, n \in N\right\}$ and $\left\{b_{n}, n \in N\right\}$ of $H$ such that $\operatorname{esssup}(H)=$ $\operatorname{esssup}\left(\left\{a_{n}, n \in N\right\}\right)$ and $\operatorname{essinf}(H)=\operatorname{essinf}\left(\left\{b_{n}, n \in N\right\}\right)$, where $N$ stands for the set of positive integers. Furthermore, if $H$ is directed upwards (downwards) then $\left\{a_{n}, n \in N\right\}$ can be chosen as nondecreasing (resp. $\left\{b_{n}, n \in N\right\}$ can be chosen as nonincreasing).

Proposition 1.3.5 below is another formulation of Proposition 1.3.4 in terms of equivalence classes, which is frequently used in random metric theory because in random metric theory we often need to distinguish random variables from their equivalence classes.

Proposition 1.3.5 [13]. Let $\bar{L}^{0}(\mathcal{F}, R)$ be the set of equivalence classes of elements in $\overline{\mathcal{L}}^{0}(\mathcal{F}, R)$, partially ordered via $\xi \leqslant \eta$ iff $\xi^{0}(\omega) \leqslant \eta^{0}(\omega) P-$ a.s., where $\xi^{0}$ and $\eta^{0}$ are arbitrarily chosen representatives of $\xi$ and $\eta$ in $\bar{L}^{0}(\mathcal{F}, R)$, respectively. Then every subset $H$ of $\bar{L}^{0}(\mathcal{F}, R)$ has a supremum, denoted by $\vee H$, and an infimum, denoted by $\wedge H$. Furthermore, there exists a countable subset $\left\{a_{n}, n \in N\right\}\left(\left\{b_{n}, n \in N\right\}\right)$ of $H$ such that $\vee H=\vee_{n \geqslant 1} a_{n}\left(\right.$ resp. $\left.\wedge H=\wedge_{n \geqslant 1} b_{n}\right)$, and $\left\{a_{n}, n \in N\right\}$ (resp. $\left\{b_{n}, n \in N\right\}$ ) can be chosen as nondecreasing (resp.,nonincreasing) if $H$ is directed upwards (downwards). Finally, $L^{0}(\mathcal{F}, R)$ as a sublattice of $\bar{L}^{0}(\mathcal{F}, R)$ is complete in the sense that every subset having an upper bound has a supremum. 
Besides, let $\xi$ and $\eta$ be two elements in $\bar{L}^{0}(\mathcal{F}, R)$. Then $\xi<\eta$ is understood as usual, namely $\xi \leqslant \eta$ and $\xi \neq \eta$. For an $\mathcal{F}$-measurable $A, \xi<\eta$ on $A$ is understood as $\xi^{0}(\omega)<\eta^{0}(\omega) P-a . s$. on $A$, where $\xi^{0}$ and $\eta^{0}$ are arbitrarily chosen representatives of $\xi$ and $\eta$, respectively.

Specially,

$$
L_{+}^{0}(\mathcal{F})=\left\{\xi \in L^{0}(\mathcal{F}, R): \xi \geqslant 0\right\}
$$

and

$$
L_{++}^{0}(\mathcal{F})=\left\{\xi \in L_{+}^{0}(\mathcal{F}): \xi>0 \text { on } \Omega\right\} .
$$

\section{Foundations}

\subsection{The central framework in random metric theory}

Basic notions in this subsection are essentially adopted from [31, 34], but the changes of some notation and convention from the theory of probabilistic metric spaces have been made as in [39] in order to make our work better known to the scholars working in other fields.

Definition 2.1.1 [31]. An ordered pair $(S,\|\cdot\|)$ is called a random normed space (briefly, an $R N$ space) over $K$ with base $(\Omega, \mathcal{F}, P)$ if $S$ is a linear space over $K$ and $\|\cdot\|$ is a mapping from $S$ to $L_{+}^{0}(\mathcal{F})$ such that the following axioms are satisfied:

$(R N-1)\|\alpha x\|=|\alpha|\|x\|, \forall \alpha \in K$ and $x \in S$;

$(R N-2)\|x+y\| \leqslant\|x\|+\|y\|, \forall x, y \in S$

$(R N-3)\|x\|=0$ implies $x=\theta$ (the null vector in $S$ ),

where $\|x\|$ is called the random norm of the vector $x$. Besides, a mapping $\|\cdot\|$ from $S$ to $L_{+}^{0}(\mathcal{F})$ is called a random seminorm on $S$ if it only satisfies $(R N-1)$ and $(R N-2)$.

In addition, if $S$ is a left module over the algebra $L^{0}(\mathcal{F}, K)$ and $\|\cdot\|$ also satisfies the following:

$(R N M-1)\|\xi x\|=|\xi|\|x\|, \forall \xi \in L^{0}(\mathcal{F}, K)$ and $x \in S$.

Then, such an $R N$ space $(S,\|\cdot\|)$ is called a random normed module (briefly, an $R N$ module) over $K$ with base $(\Omega, \mathcal{F}, P)$, such a random norm $\|\cdot\|$ is called an $L^{0}$-norm. Besides, a mapping $\|\cdot\|$ from $S$ to $L_{+}^{0}(\mathcal{F})$ is called an $L^{0}$-seminorm on $S$ if it only satisfies $(R N M-1)$ and $(R N-2)$.

Remark 2.1.1. The notion of an $R N$ module was first presented in [26], the current Definition 2.1.1 is the elaborated version of that given in [26]. Clearly, $(R N M-1)$ implies $(R N-1)$. It should also be mentioned that the notions of $L^{0}$-seminorm and $L^{0}$-norm have been known for ten years since the notion of an $R N$ module was given in [31], and the two notions were frequently employed in our previous papers [34, 35, 42, 43]. Recently, motivated by financial applications, the two notions of an $L^{0}$-seminorm and $L^{0}$-norm were rediscovered in $[15,56]$ and the notion of an $L^{0}$-normed module presented in $[15,56]$ is exactly the notion of a random normed module, see [39] for details. Finally, the notion of an original $R N$ space was first introduced in [63] and the current notion of an $R N$ space was first given in [31] as a new version of the notion of an original $R N$ space. By the way, if, in the definition of an $R N$ space over $R$ with base $(\Omega, \mathcal{F}, P), L^{0}(\mathcal{F}, R)$ is replaced by a complete vector lattice $Z$ and $L_{+}^{0}(\mathcal{F})$ by $Z^{+}:=\{z \in Z \mid z \geqslant 0\}$, then the space obtained in such a way is called a space normed with the elements of $Z$, which was first introduced in [54]. Clearly, an $R N$ space is a special space normed with the elements of a complete vector lattice, but [54] has 
never been mentioned in the literature of the theory of probabilistic metric spaces perhaps since Kantorovic[54] did not give the notion of an $R N$ space, in particular, since Kantorovic[54] did not involve any discussion of randomness.

Definition 2.1.2 [31]. An ordered pair $(S,\langle\cdot, \cdot\rangle)$ is called a random inner product space (briefly, an RIP space) over $K$ with base $(\Omega, \mathcal{F}, P)$ if $S$ is a linear space over $K$ and $\langle\cdot, \cdot\rangle$ is a mapping from $S \times S$ to $L^{0}(\mathcal{F}, K)$ such that the following axioms are satisfied:

$(\mathrm{RIP}-1)\langle x, x\rangle \in L_{+}^{0}(\mathcal{F}), \forall x \in S$, and $\langle x, x\rangle=0$ iff $x=\theta$;

(RIP-2) $\langle\alpha x, y\rangle=\alpha\langle x, y\rangle, \forall \alpha \in K$ and $x, y \in S$

(RIP-3) $\langle x, y\rangle=\overline{\langle y, x\rangle}, \forall x, y \in S$, where $\overline{\langle y, x\rangle}$ denotes the complex conjugate of $\langle y, x\rangle$;

(RIP-4) $\langle x+y, z\rangle=\langle x, z\rangle+\langle y, z\rangle, \forall x, y, z \in S$,

where $\langle x, y\rangle$ is called the random inner product from $x$ to $y$.

In addition, if $S$ is a left module over the algebra $L^{0}(\mathcal{F}, K)$ and the following axiom is also satisfied:

$(R I P M-1)\langle\xi x, y\rangle=\xi\langle x, y\rangle, \forall \xi \in L^{0}(\mathcal{F}, K)$ and $x, y \in S$,

then such an RIP space $(S,\langle\cdot, \cdot\rangle)$ is called a random inner product module (briefly, an RIP module) over $K$ with base $(\Omega, \mathcal{F}, P)$, and $\langle\cdot, \cdot\rangle$ is called an $L^{0}$-inner product on $S$.

Proposition 2.1.1 [31]. Let $(S,\langle\cdot, \cdot\rangle)$ be an RIP space over $K$ with base $(\Omega, \mathcal{F}, P)$. Define $\|\cdot\|: S \rightarrow L_{+}^{0}(\mathcal{F})$ by $\|x\|=\sqrt{\langle x, x\rangle}, \forall x \in S$. Then we have the following statements:

(1) (Cauchy-Schwartz inequality) $|\langle x, y\rangle| \leqslant\|x\|\|y\|, \forall x, y \in S$;

(2) $(S,\|\cdot\|)$ is an $R N$ space over $K$ with base $(\Omega, \mathcal{F}, P)$, and is an $R N$ module if $(S,\langle\cdot, \cdot\rangle)$ is an RIP module;

(3) A random norm $\|\cdot\|$ on a linear space $S$ is derivable from some random inner product $\langle\cdot, \cdot\rangle$ on $S$ as above iff $\|\cdot\|$ satisfies the parallelogram law, namely $\|x+y\|^{2}+\|x-y\|^{2}=2\|x\|^{2}+2\|y\|^{2}, \forall x, y \in S$.

Definition 2.1.3 [34]. $\quad$ An ordered pair $(S, \mathcal{P})$ is called a random locally convex space over $K$ with base $(\Omega, \mathcal{F}, P)$ if $S$ is a linear space over $K$ and $\mathcal{P}$ is a family of random seminorms on $S$ such that the following axiom is satisfied:

(1) $\vee\{\|x\|:\|\cdot\| \in \mathcal{P}\}=0$ iff $x=\theta$.

In addition, if $S$ is a left module over the algebra $L^{0}(\mathcal{F}, K)$ and each $\|\cdot\|$ in $\mathcal{P}$ is an $L^{0}$-seminorm, then such a random locally convex space is called a random locally convex module over $K$ with base $(\Omega, \mathcal{F}, P)$.

Remark 2.1.2. The terminologies "random locally convex modules and random locally convex spaces" were first employed in [45], and they were called "random seminormed modules and random seminormed spaces" in our previous papers $[28,34,43,51]$. When $\mathcal{P}$ reduces to a singleton $\{\|\cdot\|\}$, then a random locally convex module $(S, \mathcal{P})$ is exactly an $R N$ module $(S,\|\cdot\|)$. Clearly, $L^{0}(\mathcal{F}, K)$ is an $R N$ module over $K$ with base $(\Omega, \mathcal{F}, P)$ endowed with the $L^{0}$-norm $\|\cdot\|$ defined by $\|x\|=$ $|x|, \forall x \in L^{0}(\mathcal{F}, K)$, where $|x|$ is the equivalence class of the composite function $\left|x^{0}\right|: \Omega \rightarrow[0,+\infty)$ defined by $\left|x^{0}\right|(\omega)=\left|x^{0}(\omega)\right|, \forall \omega \in \Omega$, while $x^{0}$ is an arbitrarily chosen representative of $x$. 


\subsection{Topological structures}

The $(\epsilon, \lambda)$-topology was first introduced by Schweizer and Sklar in 1961 for probabilistic metric spaces, see [63] for details. It is naturally and frequently used in probability theory since the $(\epsilon, \lambda)$ topology for $L^{0}(\mathcal{F}, K)$ is exactly the well known topology of convergence in probability $P$. Definition 2.2.2 below merely employed the idea of Schweizer and Sklar's introduction of the $(\epsilon, \lambda)$-topology for probabilistic metric spaces.

In the sequel, for a random locally convex space $(S, \mathcal{P})$ with base $(\Omega, \mathcal{F}, P)$ and for each finite subfamily $Q$ of $\mathcal{P},\|\cdot\|_{Q}: S \rightarrow L_{+}^{0}(\mathcal{F})$ always denotes the random seminorm of $S$ defined by $\|x\|_{Q}=\vee\{\|x\| \mid\|\cdot\| \in Q\}, \forall x \in S$, and $\mathcal{F}(\mathcal{P})$ the set of finite subfamilies of $\mathcal{P}$.

Besides, we always follow the convention from random metric theory: for each $A \in \mathcal{F}, I_{A}$ stands for the characteristic function of $A$, and $\tilde{I}_{A}$ its equivalence class.

Definition 2.2.1. $\quad$ Let $(S, \mathcal{P})$ be a random locally convex space over $K$ with base $(\Omega, \mathcal{F}, P)$. For each countable subset $\left\{Q_{n} \mid n \in N\right\}$ of $\mathcal{F}(\mathcal{P})$ and each countable partition $\left\{A_{n} \mid n \in N\right\}$ of $\Omega$ to $\mathcal{F}$ (namely each $A_{n} \in \mathcal{F}, \bigcup_{n \geqslant 1} A_{n}=\Omega$, and $A_{n} \cap A_{m}=\emptyset$ when $m \neq n$ ), the random seminorm $\|\cdot\|: S \rightarrow L_{+}^{0}(\mathcal{F})$ defined by $\|x\|=\sum_{n \geqslant 1} \tilde{I}_{A_{n}}\|x\|_{Q_{n}}, \forall x \in S$, is called a countable concatenation in $\mathcal{P}$. Denote by $\mathcal{P}_{c c}$ the set of countable concatenations in $\mathcal{P}$. $\mathcal{P}$ is said to have the countable concatenation property if $\mathcal{P}_{c c}=\mathcal{P}$.

Remark 2.2.1. The countable concatenation property for the family of $L^{0}$-seminorms was first introduced in [15]. Readers can easily see that $\mathcal{P}_{c c}$ plays the same role as the saturation of $\mathcal{P}$ introduced in [41]. Clearly, $\left(S, \mathcal{P}_{c c}\right)$ is also a random locally convex space.

Definition 2.2.2 $[28,34]$. Let $(S, \mathcal{P})$ be a random locally convex space over $K$ with base $(\Omega, \mathcal{F}, P)$. For any positive real numbers $\epsilon$ and $\lambda$ such that $0<\lambda<1$, and any $Q \in \mathcal{F}(\mathcal{P})$, let $N_{\theta}(Q, \epsilon, \lambda)=$ $\left\{x \in S \mid P\left\{\omega \in \Omega \mid\|x\|_{Q}(\omega)<\epsilon\right\}>1-\lambda\right\}$, then $\left\{N_{\theta}(Q, \epsilon, \lambda) \mid Q \in \mathcal{F}(\mathcal{P}), \epsilon>0,0<\lambda<1\right\}$ is easily verified to be a local base at the null vector $\theta$ of some Hausdorff linear topology. The linear topology is called the $(\epsilon, \lambda)$-topology introduced by $\mathcal{P}$.

From now on, the $(\epsilon, \lambda)$-topology for each random locally convex space is always denoted by $\mathcal{T}_{\epsilon, \lambda}$ whenever no confusion occurs.

Proposition 2.2.1 [34]. $\quad$ Let $(S, \mathcal{P})$ be a random locally convex space over $K$ with base $(\Omega, \mathcal{F}, P)$. Then we have the following statements:

(1) The $(\epsilon, \lambda)$-topology induced by $\mathcal{P}$ is the same as the one induced by $\mathcal{P}_{c c}$;

(2) The $(\epsilon, \lambda)$-topology for $L^{0}(\mathcal{F}, K)$ is exactly the topology of convergence in probability $P$, and $\left(L^{0}(\mathcal{F}, K), \mathcal{T}_{\epsilon, \lambda}\right)$ is a topological algebra over $K$;

(3) If $(S, \mathcal{P})$ is a random locally convex module, then $\left(S, \mathcal{T}_{\epsilon, \lambda}\right)$ is a topological module over the topological algebra $L^{0}(\mathcal{F}, K)$;

(4) A net $\left\{x_{\delta}, \delta \in \Gamma\right\}$ converges in the $(\epsilon, \lambda)$-topology to some $x$ in $S$ iff for each $\|\cdot\| \in \mathcal{P}$ $\left\{\left\|x_{\delta}-x\right\|, \delta \in \Gamma\right\}$ converges in probability $P$ to 0 .

The following locally $L^{0}$-convex topology is easily seen to be much stronger than the $(\epsilon, \lambda)$ topology, and was first introduced by Filipović et al. in [15] for random locally convex modules.

Definition 2.2.3 [15]. $\quad$ Let $(S, \mathcal{P})$ be a random locally convex space over $K$ with base $(\Omega, \mathcal{F}, P)$. 
For any $Q \in \mathcal{F}(\mathcal{P})$ and $\epsilon \in L_{++}^{0}(\mathcal{F})$, let

$$
N_{\theta}(Q, \epsilon)=\left\{x \in S \mid\|x\|_{Q} \leqslant \epsilon\right\} .
$$

A subset $G$ of $S$ is called $\mathcal{T}_{c}$-open if for each $x \in G$ there exists some $N_{\theta}(Q, \epsilon)$ such that $x+N_{\theta}(Q, \epsilon) \subset$ $G, \mathcal{T}_{c}$ denotes the family of $\mathcal{T}_{c}$-open subsets of $S$. Then it is easy to see that $\left(S, \mathcal{T}_{c}\right)$ is a Hausdorff topological group with respect to the addition on $S . \mathcal{T}_{c}$ is called the locally $L^{0}$-convex topology induced by $\mathcal{P}$.

Remark 2.2.2. $\quad \mathcal{T}_{c}$ as in Definition 2.2 .3 is not necessarily a linear topology. As shown in [15], the locally $L^{0}$-convex topology $\mathcal{T}_{c}$ for the algebra $L^{0}(\mathcal{F}, K)$ are only a topological ring in general, for example, the mapping $\alpha \rightarrow \alpha \cdot 1$ may be not continuous in general, where 1 denotes the multiplication unit element of $L^{0}(\mathcal{F}, K)$.

From now on, the locally $L^{0}$-convex topology for each random locally convex space is always denoted by $\mathcal{T}_{c}$ whenever no confusion exists.

Proposition 2.2.2 [15]. $\quad$ Let $(S, \mathcal{P})$ be a random locally convex module over $K$ with base $(\Omega, \mathcal{F}, P)$. Then $\left(S, \mathcal{T}_{c}\right)$ is a Hausdorff topological module over the topological ring $\left(L^{0}(\mathcal{F}, K), \mathcal{T}_{c}\right)$ and $\left\{N_{\theta}(Q, \epsilon) \mid Q \in\right.$ $\left.\mathcal{F}(\mathcal{P}), \epsilon \in L_{++}^{0}(\mathcal{F})\right\}$ is just a local base at $\theta$ of $\mathcal{T}_{c}$.

Topological modules over the topological algebra $\left(\left(L^{0}(\mathcal{F}, K), \mathcal{T}_{\epsilon, \lambda}\right)\right.$ were earlier and deeply studied in [43], whereas topological modules over the topological ring $\left(L^{0}(\mathcal{F}, K), \mathcal{T}_{c}\right)$ were only recently studied in [15]. To introduce an important result of [15], we first give Definition 2.2.4 below, which was independently introduced in [15] and [41], in particular, the notion of an $L^{0}$-convex set almost occurred in all our previous work, see e.g., $[28,31,42,44,45]$ in the name of an $M$-convex set.

Definition 2.2.4 [15, 41]. Let $S$ be a left module over the algebra $L^{0}(\mathcal{F}, K)$ and $A$ a subset of $S$. Then $A$ is called $L^{0}$-convex if $\xi x+\eta y \in A$ for any $x$ and $y$ in $A$ and for any $\xi$ and $\eta$ in $L_{+}^{0}(\mathcal{F})$ such that $\xi+\eta=1$. A is called $L^{0}$-absorbent if for each $x \in S$ there exists some $\xi \in L_{++}^{0}(\mathcal{F})$ such that $x \in \xi A:=\{\xi a \mid a \in A\} . A$ is called $L^{0}$-balanced if $\xi x \in A$ for any $x \in A$ and $\xi \in L^{0}(\mathcal{F}, K)$ such that $|\xi| \leqslant 1$.

Definition 2.2.5 [15]. A topological module $(S, \mathcal{T})$ over the topological ring $\left(L^{0}(\mathcal{F}, K), \mathcal{T}_{c}\right)$ is called a locally $L^{0}$-convex module over $K$ with base $(\Omega, \mathcal{F}, P)$ if there is a local base $\mathcal{U}_{\theta}$ at $\theta$ for $\mathcal{T}$ such that each element in $\mathcal{U}_{\theta}$ is $L^{0}$-convex, $L^{0}$-absorbent and $L^{0}$-balanced.

Clearly, for each random locally convex module $(S, \mathcal{P}),\left(S, \mathcal{T}_{c}\right)$ is a Hausdorff locally $L^{0}$-convex module. What is important is that Filipovic et al. made use of the notion of a gauge function to obtain the following:

Proposition 2.2.3 [15]. A topological module $(S, \mathcal{T})$ over the topological ring $\left(L^{0}(\mathcal{F}, K), \mathcal{T}_{c}\right)$ is a locally $L^{0}$-convex module iff $\mathcal{T}$ can be induced by a family of $L^{0}$-seminorms on $S$.

Thus the theory of Hausdorff locally $L^{0}$-convex modules amounts to that of random locally convex modules endowed with the locally $L^{0}$-convex topology, namely the locally $L^{0}$-convex topology perfectly matches the family of $L^{0}$-seminorms. Besides, the locally $L^{0}$-convex topology has one more advantage: it is strong enough to play a crucial role in continuity and subdifferentiability theorems for lower semicontinuous $L^{0}$-valued proper functions, see Section 7 for details. On the other hand, it is also too strong to make many basic theorems concerning separation and duality 
in locally $L^{0}$-convex modules valid unless these modules possess a new countable concatenation property as introduced in [39] and Definition 2.2.6 below, see Sections 5 and 7 for details. Finally, the locally $L^{0}$-convex topology does not possess some pleasant properties as in Proposition 2.2.4 below, but the $(\epsilon, \lambda)$-topology can complement these drawbacks of the locally $L^{0}$-convex topology. Consequently, in the long run, the two kinds of topologies should be simultaneously considered in the future development of random locally convex modules, just as pointed out in [38].

Let $(S,\|\cdot\|)$ be an $R N$ module over $K$ with base $(\Omega, \mathcal{F}, P)$ and $p$ an extended positive number such that $1 \leqslant p \leqslant+\infty$. Define $\|\cdot\|_{p}: S \rightarrow[0,+\infty]$ as follows for $x$ in $S$ :

$$
\|x\|_{p}= \begin{cases}\left(\int_{\Omega}(\|x\|)^{p} d P\right)^{\frac{1}{p}}, & \text { when } 1 \leqslant p<+\infty, \\ \text { the } P \text {-essential supremum of }\|x\|, & p=+\infty\end{cases}
$$

Denote $\left\{x \in S \mid\|x\|_{p}<+\infty\right\}$ by $L^{p}(S)$, then $\left(L^{p}(S),\|\cdot\|_{p}\right)$ is a normed space over $K$, and a Banach space if $S$ is $\mathcal{T}_{\epsilon, \lambda}$-complete. It is easy to see that $L^{p}(S)$ is just the ordinary Lebesgue-Bochner function space $L^{p}(\mathcal{F}, B)$ when $S$ is the $R N$ module $L^{0}(\mathcal{F}, B)$ of equivalence classes of $\mathcal{F}$-random variables from $(\Omega, \mathcal{F}, P)$ to a normed space $B$ (see Section 2.3 below).

Although one can define $L^{p}(S)$ for an $R N$ space $(S,\|\cdot\|), L^{p}(S)$ may be trivial, namely $L^{p}(S)=$ $\{\theta\}$. Proposition 2.2.4 below shows that this would not occur for an $R N$ module $(S,\|\cdot\|)$ and also reflects the importance of the $(\epsilon, \lambda)$-topology.

Proposition 2.2.4 $[30,32,42,45] . \quad L^{p}(S)$ is $\mathcal{T}_{\epsilon, \lambda}$-dense in every $R N$ module $(S,\|\cdot\|)$ for each $p$ such that $1 \leqslant p \leqslant+\infty$.

Definition 2.2.6 below, namely the notion of the countable concatenation property of a left module over the algebra $L^{0}(\mathcal{F}, K)$ was first introduced in [39], which is different from either of the two kinds of countable concatenation properties introduced in [15]. In fact, the two kinds of countable concatenation properties in the sense of [15] are essentially identical, namely the countable concatenation property of a family of $L^{0}$-seminorms as introduced in Definition 2.2.1.

Let $S$ be a left module over the algebra $L^{0}(\mathcal{F}, K)$. For any given countable subset $\left\{x_{n}, n \in N\right\}$ and any given countable partition $\left\{A_{n}, n \in N\right\}$ of $\Omega$ to $\mathcal{F}$, the countable formal sum $\sum_{n \geqslant 1} \tilde{I}_{A_{n}} x_{n}$ is not defined in general, where we call it a countable concatenation. If $\left\{x_{n}, n \in N\right\}$ is contained in a subset $G$ of $S$, then the countable concatenation $\sum_{n \geqslant 1} \tilde{I}_{A_{n}} x_{n}$ is called a countable concatenation from $G$. For any two countable concatenation $\sum_{n \geqslant 1} \tilde{I}_{A_{n}} x_{n}$ and $\sum_{n \geqslant 1} \tilde{I}_{B_{n}} y_{n}$, we say that they are equal if $\tilde{I}_{A_{i} \cap B_{j}} x_{i}=\tilde{I}_{A_{i} \cap B_{j}} y_{j}$ for any $i$ and $j$ in $N$. Finally, we say that a countable concatenation $\sum_{n \geqslant 1} \tilde{I}_{A_{n}} x_{n}$ is well defined if there exists $x \in S$ such that $\tilde{I}_{A_{n}} x=\tilde{I}_{A_{n}} x_{n}$ for any $n \in N$. In this paper, we always make the convention for a module $S$ over the algebra $L^{0}(\mathcal{F}, K)$ : for any two elements $x$ and $y$ in $S$, if there exists a countable partition $\left\{A_{n}, n \in N\right\}$ of $\Omega$ to $\mathcal{F}$ such that $\tilde{I}_{A_{n}} x=\tilde{I}_{A_{n}} y$ for each $n \in N$, then $x=y$. It is easy to see that any random locally convex module $(S, \mathcal{P})$ satisfies the above convention.

Definition 2.2.6 [39]. Let $S$ be a left module over the algebra $L^{0}(\mathcal{F}, K)$ and $G$ a subset of $S$. The set of countable concatenations from $G$, denoted by $G_{c c}$, is called the countable concatenation hull of $G$. If $G_{c c}=G$, then $G$ is called having the countable concatenation property, namely $G$ is closed under the countable concatenation operation, that is to say, for any countable concatenation 
$\sum_{n \geqslant 1} \tilde{I}_{A_{n}} x_{n}$ from $G$, there exists $x$ in $G$ such that $\tilde{I}_{A_{n}} x=\tilde{I}_{A_{n}} x_{n}$, for any $n \in N$. Specially, if $S_{c c}=S$, then $S$ is called having the countable concatenation property.

Now, we can say that the countable concatenation property is ubiquitous in random metric theory: for any $\mathcal{T}_{\epsilon, \lambda}$-complete random locally convex module $(S, \mathcal{P}), S$ has the countable concatenation property; The random conjugate space $E_{\epsilon, \lambda}^{*}$ (see Section 2.4) of a random locally convex module under the $(\epsilon, \lambda)$-topology also has the countable concatenation property; For all random normed modules $(S,\|\cdot\|)$ occurring in random analysis and the study of conditional risk measures (see Section 2.3), $S$ has the countable concatenation property. It turned out that the deep advances in the theory of $R N$ modules together with their random conjugate spaces[27, 29, 30, 39, 41, 42, 45, 46] just benefited from the countable concatenation property.

Propositions 2.2.5 and 2.2.6 below are of fundamental importance in the theory of random locally convex modules.

Proposition 2.2.5 [39]. Let $(S, \mathcal{P})$ be a random locally convex module and $A$ a subset having the countable concatenation property of $S$. Then $\bar{A}_{c}=\bar{A}_{\epsilon, \lambda}$, where $\bar{A}_{c}$ and $\bar{A}_{\epsilon, \lambda}$ stand for the $\mathcal{T}_{c}$-closure and $\mathcal{T}_{\epsilon, \lambda}$-closure of $A$, respectively. Specially, $A$ is $\mathcal{T}_{c}$-closed iff $A$ is $\mathcal{T}_{\epsilon, \lambda}$-closed.

Proposition 2.2.6 [39]. $\quad$ Let $(S, \mathcal{P})$ be a random locally convex module. Then $S$ is $\mathcal{T}_{c}$-complete if $S$ is $\mathcal{T}_{\epsilon, \lambda}$-complete. Furthermore, if $S$ has the countable concatenation property, then $S$ is $\mathcal{T}_{c}$-complete iff $S$ is $\mathcal{T}_{\epsilon, \lambda}$-complete.

\subsection{Important examples}

Let us first recall from [57]: Let $(B,\|\cdot\|)$ be a normed space over $K$. Then a mapping $x^{0}$ : $(\Omega, \mathcal{F}, P) \rightarrow(B,\|\cdot\|)$ is called a $B$-valued $\mathcal{F}$-random variable on $\Omega$ if it is the pointwise limit of a sequence of simple $B$-valued $\mathcal{F}$-measurable mapping on $\Omega$. Further, $B^{\prime}$ denotes the classical conjugate space of $B$, then a mapping $q^{0}:(\Omega, \mathcal{F}, P) \rightarrow B^{\prime}$ is called a $\mathrm{w}^{*}$-random variable if the composite function $\left\langle b, q^{0}\right\rangle: \Omega \rightarrow K$ defined by $\left\langle b, q^{0}\right\rangle(\omega)=\left\langle b, q^{0}(\omega)\right\rangle:=\left(\left(q^{0}\right)(\omega)\right)(b), \forall \omega \in \Omega$, is a $K$-valued random variable for any given $b \in B$. For a $\mathrm{w}^{*}$-random variable $q^{0}$, the nonnegative function $\left\|q^{0}\right\|: \Omega \rightarrow[0,+\infty)$ defined by $\left\|q^{0}\right\|(\omega)=\left\|q^{0}(\omega)\right\|, \forall \omega \in \Omega$, is not necessarily $\mathcal{F}$-measurable, but $\xi_{q^{0}}:=\operatorname{esssup}\left\{\left|\left\langle b, q^{0}\right\rangle\right| \mid b \in B\right.$ and $\left.\|b\| \leqslant 1\right\}$ is always a nonnegative real-valued $\mathcal{F}$-measurable function on $\Omega$.

Example 2.3.1 [27]. Denote by $L^{0}(\mathcal{F}, B)$ the linear space of equivalence classes of $B$-valued $\mathcal{F}$ random variables on $\Omega$. The module multiplication operation $\cdot: L^{0}(\mathcal{F}, K) \times L^{0}(\mathcal{F}, B) \rightarrow L^{0}(\mathcal{F}, B)$ is defined by $\xi x=$ the equivalence class of $\xi^{0} x^{0}$, where $\xi^{0}$ and $x^{0}$ are the respective arbitrarily chosen representatives of $\xi \in L^{0}(\mathcal{F}, K)$ and $x \in L^{0}(\mathcal{F}, B)$, and $\left(\xi^{0} x^{0}\right)(\omega)=\xi^{0}(\omega) \cdot x^{0}(\omega), \forall \omega \in \Omega$. Furthermore, the mapping $\|\cdot\|: L^{0}(\mathcal{F}, B) \rightarrow L_{+}^{0}(\mathcal{F})$ is defined by $\|x\|=$ the equivalence class of $\left\|x^{0}\right\|, \forall x \in L^{0}(\mathcal{F}, B)$, where $x^{0}$ is as above. Then it is easy to see that $\left(L^{0}(\mathcal{F}, B),\|\cdot\|\right)$ is an $R N$ module over $K$ with base $(\Omega, \mathcal{F}, P)$.

Example 2.3.2 [27, 33]. Denote by $L^{0}\left(\mathcal{F}, B^{\prime}, \mathrm{w}^{*}\right)$ the linear space of $\mathrm{w}^{*}$-equivalence classes of $B^{\prime}$ valued $\mathrm{w}^{*}$-random variables on $\Omega$. The module multiplication operation on $L^{0}\left(\mathcal{F}, B^{\prime}, \mathrm{w}^{*}\right)$ is similarly defined as in the case of $L^{0}(\mathcal{F}, B)$. Furthermore, the mapping $\|\cdot\|: L^{0}\left(\mathcal{F}, B^{\prime}, \mathrm{w}^{*}\right) \rightarrow L_{+}^{0}(\mathcal{F})$ is defined by $\|q\|=$ the equivalence class of $\xi_{q^{0}}$, where $q^{0}$ is an arbitrarily chosen representative of 
$q \in L^{0}\left(\mathcal{F}, B^{\prime}, \mathrm{w}^{*}\right)$. Then it is easy to see that $\left(L^{0}\left(\mathcal{F}, B^{\prime}, \mathrm{w}^{*}\right),\|\cdot\|\right)$ is an $R N$ module over $K$ with base $(\Omega, \mathcal{F}, P)$.

Example 2.3.3 [39]. Let $(S,\|\cdot\|)$ be an $R N$ module over $K$ with base $(\Omega, \mathcal{E}, P)$ and $\mathcal{F}$ a sub $\sigma$-algebra of $\mathcal{E}$. $\||\cdot|\|_{p}: S \rightarrow \bar{L}_{+}^{0}(\mathcal{F})$ is defined as follows for $x \in S$ and $p \in[1,+\infty]$ :

$$
\|x\|_{p}= \begin{cases}{\left[E\left(\|x\|^{p} \mid \mathcal{F}\right)\right]^{\frac{1}{p}},} & \text { when } 1 \leqslant p<+\infty \\ \wedge\left\{\xi \in \bar{L}_{+}^{0}(\mathcal{F}) \mid \xi \geqslant\|x\|\right\}, & p=+\infty\end{cases}
$$

where $E\left(\|x\|^{p} \mid \mathcal{F}\right)=\lim _{n \rightarrow \infty} E\left(\|x\|^{p} \wedge n \mid \mathcal{F}\right)$ denotes the extended conditional expectation. Let $L_{\mathcal{F}}^{p}(S)=\left\{x \in S\left|\|x \mid\|_{p} \in L_{+}^{0}(\mathcal{F})\right\}\right.$, then it is easy to see that $\left(L_{\mathcal{F}}^{p}(S),\||\cdot|\|_{p}\right)$ is an $R N$ module over $K$ with base $(\Omega, \mathcal{F}, P)$.

The method to construct Example 2.3.3 comes from [15] where an extremely important $R N$ module $L_{\mathcal{F}}^{p}(\mathcal{E})$ was constructed. Since $L_{\mathcal{F}}^{p}(\mathcal{E})$ has been used as the model space for conditional risk measures in [16], it should be given as follows:

Example 2.3.4 [15]. $\quad$ Let $(\Omega, \mathcal{E}, P)$ be a probability space and $\mathcal{F}$ a sub $\sigma$-algebra of $\mathcal{E}$. Take $S=L^{0}(\mathcal{E}, R)$ in Example 2.3.3, then $L_{\mathcal{F}}^{p}(S)$ is exactly $L_{\mathcal{F}}^{p}(\mathcal{E})$ as constructed in [15].

\subsection{Random conjugate spaces and Hahn-Banach extension theorems for random linear functionals}

Definition 2.4.1 [33]. $\quad$ Let $S$ be a linear space over $K$. Then a linear operator from $S$ to $L^{0}(\mathcal{F}, K)$ is called a random linear functional on $S$. Furthermore if $S$ is a left module over the algebra $L^{0}(\mathcal{F}, K)$, then a module homomorphism from $S$ to $L^{0}(\mathcal{F}, K)$ is called an $L^{0}$-linear function.

Definition 2.4.2 [39]. Let $S$ be a real linear space. A mapping f from $S$ to $L^{0}(\mathcal{F}, R)$ is called a random sublinear functional on $S$ if the following are satisfied:

(1) $f(\alpha x)=\alpha f(x), \forall \alpha \geqslant 0$ and $x \in S$;

(2) $f(x+y) \leqslant f(x)+f(y), \forall x, y \in S$.

Furthermore, if $S$ is a left module over the algebra $L^{0}(\mathcal{F}, R)$, then a mapping $f$ from $S$ to $L^{0}(\mathcal{F}, R)$ is called $L^{0}$-sublinear function on $S$ if it satisfies the above (2) and the following:

(3) $f(\xi x)=\xi f(x), \forall \xi \in L_{+}^{0}(\mathcal{F})$ and $x \in S$.

Definition 2.4.3 [31]. Let $(S,\|\cdot\|)$ be an $R N$ space over $K$ with base $(\Omega, \mathcal{F}, P)$. A random linear functional $f: S \rightarrow L^{0}(\mathcal{F}, K)$ is called a.s. bounded if there exists some $\xi \in L_{+}^{0}(\mathcal{F})$ such that $|f(x)| \leqslant \xi\|x\|, \forall x \in S$. Denote by $S^{*}$ the set of a.s. bounded random linear functionals on $S$. The module multiplication operation $\cdot: L^{0}(\mathcal{F}, K) \times S^{*} \rightarrow S^{*}$ is defined by $(\xi f)(x)=\xi(f(x)), \forall \xi \in$ $L^{0}(\mathcal{F}, K), f \in S^{*}$ and $x \in S$, and the mapping $\|\cdot\|^{*}: S^{*} \rightarrow L_{+}^{0}(\mathcal{F})$ is defined by $\|f\|^{*}=\wedge\{\xi \in$ $\left.L_{+}^{0}(\mathcal{F})|| f(x) \mid \leqslant \xi\|x\|, \forall x \in S\right\}$. It is easy to see that $\left(S^{*},\|\cdot\|^{*}\right)$ is an $R N$ module over $K$ with base $(\Omega, \mathcal{F}, P)$, called the random conjugate space of $(S,\|\cdot\|)$.

Let $\Omega=[0,1], \mathcal{F}$ be the $\sigma$-algebra of Lebesgue measurable subsets of $[0,1]$ and $P$ be the Lebesgue measure, then it is well known that there is no nontrivial continuous linear functional on $\left(L^{0}(\mathcal{F}, K), \mathcal{T}_{\epsilon, \lambda}\right)$. But $L^{0}(\mathcal{F}, K)$ always has its random conjugate space (in fact, $\left(L^{0}(\mathcal{F}, K)\right)^{*}=L^{0}(\mathcal{F}, K)$, see Section 3), further, Proposition 2.4.3 below justifies the theory of random conjugate spaces. 
Proposition 2.4.1 below was first given in [24] in the context of random linear functionals, whose proof was merely a copy of the Hahn-Banach theorem for real linear functionals by noticing the order completeness of $L^{0}(\mathcal{F}, R)$. In fact, Proposition 2.4.1 is known as a special case of more general results in $[5,67]$.

Proposition 2.4.1. Let $S$ be a real linear space, $M \subset S$ a subspace, $f: M \rightarrow L^{0}(\mathcal{F}, R)$ a random linear functional and $p: S \rightarrow L^{0}(\mathcal{F}, R)$ a random sublinear functional such that $f(x) \leqslant p(x), \forall x \in$ $M$. Then there exists a random linear functional $g: S \rightarrow L^{0}(\mathcal{F}, R)$ such that $g$ extends $f$ and $g(x) \leqslant p(x), \forall x \in S$.

Proposition 2.4.2 below was essentially proved in [24] in an indirect manner, whose direct proof was recently given in [39].

Proposition 2.4.2 [24,39]. Let $S$ be a complex linear space, $M \subset S$ a subspace, $f: M \rightarrow$ $L^{0}(\mathcal{F}, C)$ a random linear functional and $p: S \rightarrow L_{+}^{0}(\mathcal{F})$ a random seminorm such that $|f(x)| \leqslant$ $p(x), \forall x \in M$. Then there exists a random linear functional $g: S \rightarrow L^{0}(\mathcal{F}, C)$ such that $g$ extends $f$ and $|g(x)| \leqslant p(x), \forall x \in S$.

Proposition 2.4.3 [24]. Let $(S,\|\cdot\|)$ be an $R N$ space over $K$ with base $(\Omega, \mathcal{F}, P), M \subset S$ a subspace and $f: M \rightarrow L^{0}(\mathcal{F}, K)$ an a.s. bounded random linear functional. Then there exists $g \in S^{*}$ such that $g$ extends $f$ and $\|f\|^{*}=\|g\|^{*}$.

Armed with the notion of a random conjugate space (namely Definition 2.4.3) and the HahnBanach theorem (namely Proposition 2.4.3), we knew that lots of basic results in classical functional analysis could be translated to $R N$ spaces. To prove the $\mathcal{T}_{\epsilon, \lambda}$-completeness of $S^{*}$ for any $R N$ space $(S,\|\cdot\|)$, we first translated the theory of bounded linear operators, which led to the subject of $[25,26,28]$.

For the sake of convenience, in the sequel we always use $\|\cdot\|$ for the random norm on any $R N$ space if no confusion produces.

Definition 2.4.4 [25, 28]. Let $E$ and $F$ be any two $R N$ spaces over $K$ with base $(\Omega, \mathcal{F}, P)$. A linear operator $T: E \rightarrow F$ is called a.s. bounded if there exists some $\xi \in L_{+}^{0}(\mathcal{F})$ such that $\|T x\| \leqslant \xi\|x\|, \forall x \in E$. Denote by $B(E, F)$ the linear space of a.s. bounded linear operators from $E$ to $F$, define $\|\cdot\|: B(E, F) \rightarrow L_{+}^{0}(\mathcal{F})$ by $\|T\|=\wedge\left\{\xi \in L_{+}^{0}(\mathcal{F}) \mid\|T x\| \leqslant \xi\|x\|, \forall x \in E\right\}, \forall T \in B(E, F)$, then $(B(E, F),\|\cdot\|)$ is an $R N$ sapce over $K$ with base $(\Omega, \mathcal{F}, P)$.

Proposition 2.4.4 [25, 28]. Let $E$ and $F$ be any two $R N$ modules over $K$ with base $(\Omega, \mathcal{F}, P)$. Then a linear operator $T: E \rightarrow F$ is a.s. bounded iff $T$ is a continuous module homomorphism from $\left(E, \mathcal{T}_{\epsilon, \lambda}\right)$ to $\left(F, \mathcal{T}_{\epsilon, \lambda}\right)$, and at which case $\|T\|=\vee\{\|T x\| \mid x \in E$ and $\|x\| \leqslant 1\}$.

Remark 2.4.1. When $\mathcal{T}_{\epsilon, \lambda}$ is replaced with $\mathcal{T}_{c}$ Proposition 2.4.4 is also true, even the proof is easier. The proof of Proposition 2.4.4 uses the fact that an $L^{0}$-convex set $A$ in an $R N$ module over $K$ with base $(\Omega, \mathcal{F}, P)$ is a.s. bounded (namely $\vee\{\|x\| \mid a \in A\} \in L_{+}^{0}(\mathcal{F})$ ) iff $A$ is $\mathcal{T}_{\epsilon, \lambda}$-bounded. An interesting observation should be made: Let $(S,\|\cdot\|)$ be an $R N$ module over $K$ with base $(\Omega, \mathcal{F}, P)$ and $A$ a subset of $S$, then $A$ is a.s. bounded iff $A$ is $\mathcal{T}_{c}$-bounded (namely for any $\mathcal{T}_{c}$-neighborhood $U$ of the null vector there exists some $\xi \in L_{++}^{0}(\mathcal{F})$ such that $\left.A \subset \xi U\right)$. This observation also holds for any random locally convex module $(S, \mathcal{P})$ with base $(\Omega, \mathcal{F}, P): A \subset S$ is $\mathcal{T}_{c}$-bounded iff $A$ is a.s. bounded, namely $\vee\{\|x\| \mid a \in A\} \in L_{+}^{0}(\mathcal{F})$ for each $\|\cdot\| \in \mathcal{P}$. 
Definition 2.4.5 [25, 28]. Let $E$ and $F$ be any two $R N$ spaces over $K$ with base $(\Omega, \mathcal{F}, P)$ and $T \in B(E, F)$. Define $T^{*}: F^{*} \rightarrow E^{*}$ by $T^{*} f(x)=f(T x), \forall x \in E$ and $f \in F^{*}$, it is very easy to prove that $T^{*} \in B\left(F^{*}, E^{*}\right)$ and $\left\|T^{*}\right\|=\|T\| . T^{*}$ is called the conjugate operator of $T$.

Definition 2.4.6 $[25,28]$. Let $(S,\|\cdot\|)$ be an $R N$ space. Define $J: S \rightarrow S^{* *}:=\left(S^{*}\right)^{*}$ by $(J(x))(f)=f(x), \forall f \in S^{*}$ and $x \in E$, then $J$ is random norm preserving. If $J$ is surjective, then $S$ is called random reflexive.

Making use of Proposition 2.4.4 Guo first proved in [28] that $B(E, F)$ is $\mathcal{T}_{\epsilon, \lambda}$-complete if $E$ and $F$ are $R N$ modules such that $F$ is $\mathcal{T}_{\epsilon, \lambda}$-complete, and hence $S^{* *}$ is always $\mathcal{T}_{\epsilon, \lambda}$-complete by noticing $S^{*}$ is an $R N$ module for an $R N$ space $S$; then Guo further proved in [28] that $B(E, F)$ always is $\mathcal{T}_{\epsilon, \lambda}$-complete for any $R N$ spaces $E$ and $F$ such that $F$ is $\mathcal{T}_{\epsilon, \lambda}$-complete. The key step in completing the proof is that Guo observed in [28] the following: if $S$ is only an $R N$ space, then the embedding mapping $J: S \rightarrow S^{* *}$ can be used to generate a $\mathcal{T}_{\epsilon, \lambda}$-complete $R N$ module from $S$. Let $M(S)$ be the $\mathcal{T}_{\epsilon, \lambda}$-closed submodule generated by $J(S)$ in $S^{* *}$, then $M(S)$ is a $\mathcal{T}_{\epsilon, \lambda}$-complete $R N$ module since $S^{* *}$ is $\mathcal{T}_{\epsilon, \lambda}$-complete.

Proposition 2.4.5 [28]. Let $E$ and $F$ be two $R N$ spaces over $K$ with base $(\Omega, \mathcal{F}, P)$ such that $F$ is $\mathcal{T}_{\epsilon, \lambda}$-complete. Define $L: B(E, F) \rightarrow B(M(E), M(F))$ by $L(T)=\left.T^{* *}\right|_{M(E)}, \forall T \in B(E, F)$, where $T^{* *}=\left(T^{*}\right)^{*}: E^{* *} \rightarrow F^{* *}$ is the conjugate operator of $T^{*}$. Then $L$ is random norm preserving and $L(B(E, F))$ is a $\mathcal{T}_{\epsilon, \lambda}$-closed subspace of $B(M(E), M(F))$. Specially $B(E, F)$ is $\mathcal{T}_{\epsilon, \lambda}$-complete.

Corollary 2.4.1 [28]. $\quad S^{*}$ is $\mathcal{T}_{\epsilon, \lambda}$-complete for any $R N$ space $S$.

When we generalize the idea of random conjugate spaces from $R N$ spaces to random locally convex spaces, historically there are two notions of a random conjugate space for a random locally convex space. It turns out that they just correspond to the locally $L^{0}$-convex topology and the $(\epsilon, \lambda)$-topology, respectively, in the context of a random locally convex module!

Definition 2.4.7 [28]. $\quad$ Let $(S, \mathcal{P})$ be a random locally convex space over $K$ with base $(\Omega, \mathcal{F}, P)$. A random linear functional $f: S \rightarrow L^{0}(\mathcal{F}, K)$ is called an a.s. bounded random linear functional of type I if there are some $\xi \in L_{+}^{0}(\mathcal{F})$ and $Q \in \mathcal{F}(\mathcal{P})$ such that $|f(x)| \leqslant \xi\|x\|_{Q}, \forall x \in S$. Denote by $S_{c}^{*}$ the linear space of a.s. bounded random linear functionals of type I on $S$, similar to Definition 2.4.3 $S_{c}^{*}$ becomes a left module over $L^{0}(\mathcal{F}, K)$, called the random conjugate space of type I of $S$.

Definition 2.4.8 [34]. $\quad$ Let $(S, \mathcal{P})$ be a random locally convex space over $K$ with base $(\Omega, \mathcal{F}, P)$. A random linear functional $f: S \rightarrow L^{0}(\mathcal{F}, K)$ is called an a.s. bounded random linear functional of type II on $S$ if there exist some $\xi \in L_{+}^{0}(\mathcal{F})$ and $\|\cdot\| \in \mathcal{P}_{c c}$ such that $|f(x)| \leqslant \xi\|x\|, \forall x \in S$ (see Section 2.2 for $\left.\mathcal{P}_{c c}\right)$. Denote by $S_{\epsilon, \lambda}^{*}$ the $L^{0}(\mathcal{F}, K)$-module of a.s. bounded random linear functionals of type II on $S$, called the random conjugate space of type II of $S$.

Proposition 2.4.6 below is essentially the simpler Lemma 2.12 of [39], which not only considerably simplifies the proof of the Hahn-Banach theorem for $L^{0}$-linear functions but also makes it easier for people to understand the topological module characterizations for $S_{c}^{*}$ and $S_{\epsilon, \lambda}^{*}$.

Proposition 2.4.6 [39]. Let $S$ be a left module over the algebra $L^{0}(\mathcal{F}, K)$ and $f: S \rightarrow L^{0}(\mathcal{F}, K)$ a random linear functional. Then, we have the following statements:

(1) If $K=R$, then $f$ is an $L^{0}$-linear function iff there exists an $L^{0}$-sublinear function $p: S \rightarrow$ $L^{0}(\mathcal{F}, R)$ such that $f(x) \leqslant p(x), \forall x \in S$; 
(2) If $K=C$, then $f$ is an $L^{0}$-linear function iff there exists an $L^{0}$-seminorm $p: S \rightarrow L_{+}^{0}(\mathcal{F})$ such that $|f(x)| \leqslant p(x), \forall x \in S$.

Corollary 2.4.2 below is easily derived from Propositions 2.4.1 and 2.4.6. Corollary 2.4.2 is known as a special case of the main results of $[5,67]$ and its proof was also given in [15]. But since not every element of $L^{0}(\mathcal{F}, R)$ has a multiplication inverse element, this brings an obstacle to one step extension in the process of the proof of Corollary 2.4.2, the complicated methods were used in $[5,15,67]$ in order to overcome this obstacle.

Corollary 2.4.2 [5, 67]. Let $S$ be a left module over the algebra $L^{0}(\mathcal{F}, R), M \subset S$ a submodule, $f: M \rightarrow L^{0}(\mathcal{F}, R)$ an $L^{0}$-linear function and $p: S \rightarrow L^{0}(\mathcal{F}, R)$ an $L^{0}$-sublinear function such that $f(x) \leqslant p(x), \forall x \in M$. Then there exists an $L^{0}$-linear function $g: S \rightarrow L^{0}(\mathcal{F}, R)$ such that $g$ extends $f$ and $g(x) \leqslant p(x), \forall x \in S$.

Corollary 2.4.3 below can be easily derived not only from Corollary 2.4.2 but also from Propositions 2.4.3 and 2.4.6.

Corollary 2.4.3 [39]. Let $S$ be a left module over the algebra $L^{0}(\mathcal{F}, C), M \subset S$ a submodule, $f: M \rightarrow L^{0}(\mathcal{F}, C)$ an $L^{0}$-linear function and $p: S \rightarrow L_{+}^{0}(\mathcal{F})$ an $L^{0}$-seminorm such that $|f(x)| \leqslant$ $p(x), \forall x \in M$. Then there is an $L^{0}$-linear function $g: S \rightarrow L^{0}(\mathcal{F}, C)$ such that $g$ extends $f$ and $|g(x)| \leqslant p(x), \forall x \in S$.

The following two propositions give the topological characterizations of $S_{c}^{*}$ and $S_{\epsilon, \lambda}^{*}$, and hence also an equivalent definition of either of $S_{c}^{*}$ and $S_{\epsilon, \lambda}^{*}$ as given in [39].

Proposition 2.4.7 [39]. $\quad$ Let $(S, \mathcal{P})$ be a random locally convex module over $K$ with base $(\Omega, \mathcal{F}, P)$ and $f: S \rightarrow L^{0}(\mathcal{F}, K)$ a random linear functional. Then $f \in S_{c}^{*}$ iff $f$ is a continuous module homomorphism from $\left(S, \mathcal{T}_{c}\right)$ to $\left(L^{0}(\mathcal{F}, K), \mathcal{T}_{c}\right)$.

Proposition 2.4.8 [34,51]. Let $(S, \mathcal{P})$ be a random locally convex module over $K$ with base $(\Omega, \mathcal{F}, P)$ and $f: S \rightarrow L^{0}(\mathcal{F}, K)$ a random linear functional. Then $f \in S_{\epsilon, \lambda}^{*}$ iff $f$ is a continuous module homomorphism from $\left(S, \mathcal{T}_{\epsilon, \lambda}\right)$ to $\left(L^{0}(\mathcal{F}, K), \mathcal{T}_{\epsilon, \lambda}\right)$.

From Propositions 2.4.7 and 2.4.8, we can now give the following topological versions of Corollaries 2.4.2 and 2.4.3.

Proposition 2.4.9[39]. $\quad$ Let $(S, \mathcal{P})$ be a random locally convex module over $K$ with base $(\Omega, \mathcal{F}, P)$ and $M \subset S$ a submodule. Then we have the following statements:

(1) every continuous module homomorphism from $\left(M, \mathcal{T}_{c}\right)$ to $\left(L^{0}(\mathcal{F}, K), \mathcal{T}_{c}\right)$ can be extended to a continuous module homomorphism from $\left(S, \mathcal{T}_{c}\right)$ to $\left(L^{0}(\mathcal{F}, K), \mathcal{T}_{c}\right)$;

(2) every continuous module homomorphism from $\left(M, \mathcal{T}_{\epsilon, \lambda}\right)$ to $\left(L^{0}(\mathcal{F}, K), \mathcal{T}_{\epsilon, \lambda}\right)$ can be extended to a continuous module homomorphism from $\left(S, \mathcal{T}_{\epsilon, \lambda}\right)$ to $\left(L^{0}(\mathcal{F}, K), \mathcal{T}_{\epsilon, \lambda}\right)$.

Proposition 2.4.10 [39]. Let $(S, \mathcal{P})$ be a random locally convex space. Then $S_{\epsilon, \lambda}^{*}=S_{c}^{*}$ if $\mathcal{P}$ has the countable concatenation property (generally, it is obvious that $S_{c}^{*} \subset S_{\epsilon, \lambda}^{*}$ ). In particular, $S_{\epsilon, \lambda}^{*}=S_{c}^{*}$ for any $R N$ space $(S,\|\cdot\|)$.

Remark 2.4.2. Before 1995, the focus of our work is on $R N$ spaces and indeed we also obtained several pleasant results, for example, Proposition 2.4.3, Proposition 2.4.5 and Corollary 2.4.1. But the results in the paper [26] and further in [28] (for example, Proposition 2.4.4) made us realize the fundamental importance of the module structure of an $R N$ module, thus after 1995 the theory of 
$R N$ modules together with their random conjugate spaces has been our concern.

\section{Representation theorems of random conjugate spaces}

Proposition 3.1 (Riesz's representation theorem) [46]. Let $(S,\langle\cdot, \cdot\rangle)$ be a $\mathcal{T}_{\epsilon, \lambda}$-complete $R I P$ module. Then there exists a unique $\pi(f)$ in $S$ for each $f \in S_{\epsilon, \lambda}^{*}$ such that $f(x)=\langle x, \pi(f)\rangle, \forall x \in S$, and $\|\pi(f)\|=\|f\|$.

Corollary 3.1 [39]. Let $(S,\langle\cdot, \cdot\rangle)$ be a $\mathcal{T}_{c}$-complete $R I P$ module such that $S$ has the countable concatenation property. Then there exists a unique $\pi(f)$ in $S$ for each $f \in S_{c}^{*}$ such that $f(x)=$ $\langle x, \pi(f)\rangle, \forall x \in S$, and $\|\pi(f)\|=\|f\|$.

Proposition 3.1 is essential and Corollary 3.1 is merely a consequence of Propositions 3.1, 2.4.10 and 2.2.6. Here, we would like to review the proof of Proposition 3.1. First, its proof is considerably different from that of the classical Riesz's representation theorem in Hilbert Spaces and the classical case only needs to utilize the orthogonal decomposition theorem, whereas the proof of Proposition 3.1 forces us to work out a countable concatenation technique in order to obtain $\pi(f)$ by means of the countable concatenation property of $S$ under $\mathcal{T}_{\epsilon, \lambda}$, and thus one should not surprise at the hypothesis on Corollary 3.1. Secondly, the following orthogonal decomposition theorem is, of course, used in the proof of Proposition 3.1.

Proposition $3.2[31,46]$. Let $(S,\langle\cdot, \cdot\rangle)$ be a $\mathcal{T}_{\epsilon, \lambda}$-complete $R I P$ module over $K$ with base $(\Omega, \mathcal{F}, \mathcal{P}), M \subset S$ a $\mathcal{T}_{\epsilon, \lambda}$-closed submodule and $M^{\perp}=\{x \in S \mid\langle x, y\rangle=0, \forall y \in M\}$. Then $S=M \oplus M^{\perp}$.

Proof. Let $x$ be any element in $S$, we prove that there exists a unique $x_{0} \in M$ such that $x-x_{0} \in$ $M^{\perp}$. First, let $d(x, M)=\wedge\{\|x-y\| \mid y \in M\}$, and for any $y_{1}, y_{2} \in M$ let $A=\left[\left\|x-y_{1}\right\| \leqslant\left\|x-y_{2}\right\|\right]$, where $A$ is the equivalence class of $A^{0}, A^{0}=\left\{\omega \in \Omega \mid\left\|x-y_{1}\right\|^{0}(\omega) \leqslant\left\|x-y_{2}\right\|^{0}(\omega)\right\},\left\|x-y_{1}\right\|^{0}$ and $\left\|x-y_{2}\right\|^{0}$ are arbitrarily chosen representatives, respectively, and $I_{A}:=\tilde{I}_{A^{0}}$. Then one can easily check that $y_{3}=I_{A} y_{1}+\left(1-I_{A}\right) y_{2} \in M$ and satisfies the relation: $\left\|x-y_{3}\right\|=\left\|x-y_{1}\right\| \wedge\left\|x-y_{2}\right\|$, which shows that $\{\|x-z\| \mid z \in M\}$ is directed downwards. Then by Proposition 1.3.5 there exists a sequence $\left\{z_{n}, n \in N\right\}$ in $M$ such that $\left\{\left\|x-z_{n}\right\| \mid n \in N\right\}$ converges to $d(x, M)$ in a nonincreasing manner. One can prove that $\left\{z_{n}, n \in N\right\}$ is a $\mathcal{T}_{\epsilon, \lambda}$-Cauchy sequence completely similar to the classical case, hence convergent to some point $x_{0}$. Further, one can also verify that $x_{0}$ is just as desired as in the classical case.

As we have seen, the proof of Proposition 3.2 is only a copy of the proof of its classical prototype, but as a spacial case of Proposition 3.2, Corollary 3.2 below used to appear in the mathematical finance literature $[21,62]$, but where its proof is indirect by a technique of converting the orthogonal decomposition problem in a special $\mathcal{T}_{\epsilon, \lambda}$-complete $R I P$ module to the corresponding problem in a Hilbert space. Now, we can give a straightforward proof.

Corollary $3.2[21,62]$. Let $\left(\Omega, \mathcal{F}_{1}, P\right)$ be a probability space, $\mathcal{F}_{0}$ a sub $\sigma$-algebra of $\mathcal{F}_{1}, y$ in $L^{0}\left(\mathcal{F}_{1}, R^{d}\right)$ (where $R^{d}$ is the d-dimensional Euclidean space), $N=\left\{x \in L^{0}\left(\mathcal{F}_{0}, R^{d}\right) \mid\langle x, y\rangle=0\right\}$ and $N^{\perp}=\left\{z \in L^{0}\left(\mathcal{F}_{0}, R^{d}\right) \mid\langle x, z\rangle=0, \forall x \in N\right\}$. Then $L^{0}\left(\mathcal{F}_{0}, R^{d}\right)=N \oplus N^{\perp}$.

Proof. Take $S=L^{0}\left(\mathcal{F}_{0}, R^{d}\right)$ and $M=N$ in Corollary 3.2, then the desired follows. 
Definition 3.1. $\quad$ Let $E$ and $F$ be any two $R N$ modules over $K$ with base $(\Omega, \mathcal{F}, P)$. A mapping $T: E \rightarrow F$ is called an isometric isomorphism between $E$ and $F$ if $T$ is a random norm preserving module isomorphism.

Let $L^{0}(\mathcal{F}, B)$ and $L^{0}\left(\mathcal{F}, B^{\prime}, \mathrm{w}^{*}\right)$ be the same as in Section 2.3. For any $x$ in $L^{0}(\mathcal{F}, B)$ and $y$ in $L^{0}\left(\mathcal{F}, B^{\prime}, \mathrm{w}^{*}\right)$, let $x^{0}$ and $y^{0}$ be any chosen representatives of $x$ and $y$, respectively, $\left\langle x^{0}, y^{0}\right\rangle: \Omega \rightarrow K$ is defined by $\left\langle x^{0}, y^{0}\right\rangle(\omega)=\left(y^{0}(\omega)\right)\left(x^{0}(\omega)\right), \forall \omega \in \Omega$. Then it is clear that $\left\langle x^{0}, y^{0}\right\rangle$ is a $K$-valued $\mathcal{F}$-random variable on $(\Omega, \mathcal{F}, P)$, and denote by $\langle x, y\rangle$ the equivalence class of $\left\langle x^{0}, y^{0}\right\rangle$. Now, for each $y \in L^{0}\left(\mathcal{F}, B^{\prime}, \mathrm{w}^{*}\right)$, define $T(y): L^{0}(\mathcal{F}, B) \rightarrow L^{0}(\mathcal{F}, K)$ by $(T(y))(x)=\langle x, y\rangle, \forall x \in L^{0}(\mathcal{F}, B)$. Then we have the following:

Proposition 3.3 [27]. Let $(\Omega, \mathcal{F}, P)$ be a complete probability space. Then $T$ defined as above is an isometric isomorphism from $L^{0}\left(\mathcal{F}, B^{\prime}, w^{*}\right)$ onto $\left(L^{0}(\mathcal{F}, B)\right)^{*}$ (namely the random conjugate space of $\left.L^{0}(\mathcal{F}, B)\right)$.

Since $L^{0}\left(\mathcal{F}, B^{\prime}\right)$ can be regarded as a submodule of $L^{0}\left(\mathcal{F}, B^{\prime}, \mathrm{w}^{*}\right)$, we should consider the following problem: When is the restriction of $T$ as in Proposition 3.3 to $L^{0}\left(\mathcal{F}, B^{\prime}\right)$ also an isometric isomorphism between $L^{0}\left(\mathcal{F}, B^{\prime}\right)$ and $\left(L^{0}(\mathcal{F}, B)\right)^{*}$ ? We have the following answer:

Proposition $3.4[27] . \quad T$ is an isometric isomorphism between $L^{0}\left(\mathcal{F}, B^{\prime}\right)$ and $\left(L^{0}(\mathcal{F}, B)\right)^{*}$ iff $B^{\prime}$ has the Radon-Nikodým property with respect to $(\Omega, \mathcal{F}, P)$.

Corollary $3.3[27]$. Let $(\Omega, \mathcal{F}, P)$ be a complete probability space. Then $B^{\prime}$ has the RadonNikodým property with respect to $(\Omega, \mathcal{F}, P)$ iff there is a $B^{\prime}$-valued random variable $\bar{q}$ for each $B^{\prime}$ valued $w^{*}$-random variable $q$ such that $q$ and $\bar{q}$ are $w^{*}$-equivalent to each other.

Proposition 3.5 below provides a pleasant connection between the random conjugate space $S^{*}$ of an $R N$ module $S$ and the classical conjugate space $\left(L^{p}(S)\right)^{\prime}$ of $\left(L^{p}(S)\right)$, which enables us to establish many difficult results, for example, Proposition 3.6 below, all the results in Section 4, and the main result of [45].

Proposition $3.5[30,34]$. Let $(S,\|\cdot\|)$ be an $R N$ module over $K$ with base $(\Omega, \mathcal{F}, P)$ and $1 \leqslant p<\infty$. Then $T:\left(L^{q}\left(S^{*}\right),\|\cdot\|_{q}\right) \rightarrow\left(L^{p}(S)\right)^{\prime}$ is an isometric isomorphism. Where $q$ is the Hölder conjugate number of $p, L^{p}(S)$ and $L^{q}\left(S^{*}\right)$ are understood as in Section 2.2, and for each $f \in L^{q}\left(S^{*}\right), T(f): L^{p}(S) \rightarrow K$ is defined by $(T(f))(g)=\int_{\Omega} f(g) d P, \forall g \in L^{p}(S)$.

Proposition 3.6 [39]. Let $(S,\|\cdot\|)$ be an $R N$ module over $K$ with base $(\Omega, \mathcal{E}, P), \mathcal{F} \subset \mathcal{E}$ a sub $\sigma$-algebra and $1 \leqslant p<\infty$ with Hölder conjugate number $q$. Then $T:\left(L_{\mathcal{F}}^{q}\left(S^{*}\right),\||\cdot|\|_{q}\right) \rightarrow\left(L_{\mathcal{F}}^{p}(S)\right)^{*}$ is isometric isomorphism, where $L_{\mathcal{F}}^{p}(S)$ and $L_{\mathcal{F}}^{q}\left(S^{*}\right)$ are understood as in Section 2.3, and for each $f \in L_{\mathcal{F}}^{q}\left(S^{*}\right) T(f): L_{\mathcal{F}}^{p}(S) \rightarrow L^{0}(\mathcal{F}, K)$ is defined by $(T(f))(g)=E(f(g) \mid \mathcal{F}), \forall g \in L_{\mathcal{F}}^{p}(S)$.

Corollary 3.4 [56]. Let $(\Omega, \mathcal{E}, P)$ be a probability space, $\mathcal{F} \subset \mathcal{E}$ a sub $\sigma$-algebra and $1 \leqslant p<+\infty$ with the Hölder conjugate number $q$. Then $T: L_{\mathcal{F}}^{q}(\mathcal{E}) \rightarrow\left(L_{\mathcal{F}}^{p}(\mathcal{E})\right)^{*}$ is an isometric isomorphism, where for each $f \in L_{\mathcal{F}}^{q}(\mathcal{E}), T(f): L_{\mathcal{F}}^{p}(\mathcal{E}) \rightarrow L^{0}(\mathcal{F}, R)$ is defined by $(T(f))(g)=E(f \cdot g \mid \mathcal{F})$, $\forall g \in L_{\mathcal{F}}^{p}(\mathcal{E})$.

Remark 3.1. The original proof of Corollary 3.4 in [56] only shows that $T$ is a module isomorphism, whereas the isometric property of $T$ was proved in [39] as a special case of Proposition 3.6. Corollary 3.4 is crucial in the dual representation of conditional risk measures; see Section 7 for details. 


\section{Characterization for random reflexivity}

According to Definition 2.4.6, if an $R N$ space is random reflexive then it has to be a both $\mathcal{T}_{c^{-}}$ complete and $\mathcal{T}_{\epsilon, \lambda}$-complete $R N$ module with the countable concatenation property since $S^{* *}$ has all the properties. In fact, as analyzed in [39], random reflexivity is independent of the special choice of two kinds of topologies, and hence we will not mention the topologies in this section.

Proposition $4.1[27] . \quad L^{0}(\mathcal{F}, B)$ is random reflexive iff $B$ is reflexive.

Proposition 4.2 [30]. An $R N$ module $S$ is random reflexive iff $L^{p}(S)$ is reflexive for any given $p$ such that $1<p<+\infty$.

Proposition 4.3 (The James Theorem) [42]. A complete $R N$ module $S$ is random reflexive iff there exists $x \in S(1)$ for each $f \in S^{*}$ such that $f(x)=\|f\|$, where $S(1)=\{y \in S \mid\|y\| \leqslant 1\}$.

Proposition 4.4 [38]. Let $(S,\|\cdot\|)$ be an $R N$ module over $K$ with base $(\Omega, \mathcal{E}, P), \mathcal{F} \subset \mathcal{E}$ a sub $\sigma$-algebra and $1<p<+\infty$. Then $L_{\mathcal{F}}^{p}(S)$ is random reflexive iff $S$ is random reflexive. Specially, $L_{\mathcal{F}}^{p}(\mathcal{E})$ is random reflexive.

\section{$5 \quad$ Hyperplane separation theorems}

For a random locally convex module $(E, \mathcal{P})$ over $K$ with base $(\Omega, \mathcal{F}, P), x \in E$ and $G \subset E$ a subset. For each $Q \in \mathcal{F}(\mathcal{P})$, let $d_{Q}^{*}(x, G)=\wedge\left\{\|x-y\|_{Q} \mid y \in G\right\}$, and $d^{*}(x, G)=\vee\left\{d_{Q}^{*}(x, G) \mid Q \in \mathcal{F}(\mathcal{P})\right\}$. Then $d^{*}(x, G) \in \bar{L}_{+}^{0}(\mathcal{F})$ in general.

Proposition $5.1[39,45]$. Let $(E, \mathcal{P})$ be a random locally convex module over $K$ with base $(\Omega, \mathcal{F}, P), x \in E, G$ a nonempty $\mathcal{T}_{\epsilon, \lambda}$-closed $L^{0}$-convex subset of $E$ such that $x \notin G$, and $\xi$ a chosen representative of $d^{*}(x, G)$. Then there exists an $f \in E_{\epsilon, \lambda}^{*}$ such that the following are satisfied:

(1) $(\operatorname{Ref})(x)>\vee\{(\operatorname{Ref})(y) \mid y \in G\}$;

(2) $(\operatorname{Ref})(x)>\vee\{(\operatorname{Ref})(y) \mid y \in G\}$ on $\{\xi>0\}$.

Proposition 5.2 [39]. Let $(E, \mathcal{P})$ be a random locally convex module over $K$ with base $(\Omega, \mathcal{F}, P)$ such that $\mathcal{P}$ has the countable concatenation property, $x \in E$ and $G$ a nonempty $\mathcal{T}_{c}$-closed $L^{0}$-convex subset of $E$ such that $x \notin G$ and $G$ has the countable concatenation property. Then there exists an $f \in E_{c}^{*}$ such that the following are satisfied:

(1) $(\operatorname{Ref})(x)>\vee\{(\operatorname{Ref})(y) \mid y \in G\}$;

(2) $(\operatorname{Ref})(x)>\vee\{(\operatorname{Ref})(y) \mid y \in G\}$ on $\{\xi>0\}$,

where $\xi$ is any chosen representative of $d^{*}(x, G)$.

Corollary $5.1[15,39]$. Let $(E, \mathcal{P})$ be a random locally convex module over $K$ with base $(\Omega, \mathcal{F}, P)$ such that $\mathcal{P}$ has the countable concatenation property, $x \in E$ and $G \subset E$ a nonempty $\mathcal{T}_{c}$-closed $L^{0}$-convex subset with the countable concatenation property. If $\tilde{I}_{A}\{x\} \cap \tilde{I}_{A} G=\emptyset$ for all $A \in \mathcal{F}$ with $P(A)>0$, then there exists an $f \in E_{c}^{*}$ and $\varepsilon \in L_{++}^{0}(\mathcal{F})$ such that $(\operatorname{Re} f)(x)>(\operatorname{Re} f)(y)+\varepsilon$ on $\Omega$ for all $y \in G$.

Remark 5.1. Corollary 5.1 improves Theorem 2.8 of [15] in that $G$ is assumed to have the countable concatenation property, whereas Theorem 2.8 of [15] did not make the hypothesis. In 
fact, we recently constructed a counterexample in [50] which shows that both Theorem 2.8 and Lemma 2.28 of [15] may be not true if the hypothesis is removed.

Now, we can give the improved version of [15, Lemma 2.28] - Proposition 5.3 below, which is very useful in improving the main results in [15], see Section 7.

Proposition 5.3 [50]. Let $(E, \mathcal{P})$ be a random locally convex module over $K$ with base $(\Omega, \mathcal{F}, P)$ such that $\mathcal{P}$ has the countable concatenation property, $x \in E$ and $G \subset E$ a $\mathcal{T}_{c}$-closed subset with the countable concatenation property. If $\tilde{I}_{A}\{x\} \cap \tilde{I}_{A} G=\emptyset$ for all $A \in \mathcal{F}$ with $P(A)>0$, then there exists an $L^{0}$-convex, $L^{0}$-absorbent and $L^{0}$-balanced $\mathcal{T}_{c^{-}}$neighborhood $U$ of $0 \in E$ such that $\tilde{I}_{A}(x+U) \cap \tilde{I}_{A}(G+U)=\emptyset$ for all $A \in \mathcal{F}$ with $P(A)>0$.

Proposition $5.4[15]$. Let $(E, \mathcal{P})$ be a random locally convex module over $K$ with base $(\Omega, \mathcal{F}, P)$, $G$ and $M$ two $L^{0}$-convex subsets of $E$ such that $G$ is also nonempty and $\mathcal{T}_{c}$-open. If $\tilde{I}_{A} G \cap \tilde{I}_{A} M=\emptyset$ for all $A \in \mathcal{F}$ with $P(A)>0$, then there exists an $f \in E_{c}^{*}$ such that $(\operatorname{Re} f)(x)<(\operatorname{Ref})(y)$ on $\Omega$ for all $x \in G$ and $y \in M$.

Remark 5.2. Proposition 5.4 is peculiar to the locally $L^{0}$-convex topology $\mathcal{T}_{c}$ since $\mathcal{T}_{\epsilon, \lambda}$ is too weak to ensure the existence of a proper, nonempty $L^{0}$-convex and $\mathcal{T}_{\epsilon, \lambda}$-open subset in a random locally convex module. By the way, we proved in [39] that Proposition 5.1 implies both Proposition 5.2 and Corollary 5.1.

\section{Random duality with respect to the locally $L^{0}$-convex topol- ogy}

The theory of a random locally convex module $(E, \mathcal{P})$ is considerably different from the theory of an ordinary locally convex space in that $\mathcal{P}$ can induce the two kinds of topologies - the locally $L^{0}$-convex topology $\mathcal{T}_{c}$ and the $(\epsilon, \lambda)$-topology $\mathcal{T}_{\epsilon, \lambda}$. Thus the theory of random duality based on the framework of a random locally convex module should have two kinds - corresponding to the above two kinds of topologies, respectively.

The theory of random duality corresponding to the $(\epsilon, \lambda)$-topology was presented and studied in $[29,34,41]$ where we could only speak of random compatible structure and random admissible structure (they were defined as a family of $L^{0}$-seminorms, respectively) rather than random compatible topology and random admissible topology because what really plays a crucial role in random duality is a family of $L^{0}$-seminorms. Thanks to the contribution made by Filipović et al. in [15], namely any locally $L^{0}$-convex topology can also be induced by a family of $L^{0}$-seminorms, which enables us to speak of random compatible and random admissible topologies under the framework of locally $L^{0}$-convex modules (namely under the framework of locally $L^{0}$-convex topologies).

The purpose of this section is to develop the theory of random duality under the locally $L^{0}$-convex topology. All the results in this section without mention of reference belong to the author, which together with some other interesting results will be published in our forthcoming joint paper [50].

Since we will consider more than one family of $L^{0}$-seminorms on a given $L^{0}(\mathcal{F}, K)$-module $E$. Given a family $\mathcal{P}$ of $L^{0}$-seminorms on the $L^{0}(\mathcal{F}, K)$-module such that $(E, \mathcal{P})$ become a random locally convex module, in this section we always use $\mathcal{P}_{c}$ and $\mathcal{P}_{\epsilon, \lambda}$ rather than $\mathcal{T}_{c}$ and $\mathcal{T}_{\epsilon, \lambda}$ as in the 
other sections for the locally $L^{0}$-convex topology and the $(\epsilon, \lambda)$-topology induced by $\mathcal{P}$, respectively. At the same time we always use $(E, \mathcal{P})_{c}^{*}$ for the random conjugate space consisting of all continuous module homomorphisms from $\left(E, \mathcal{P}_{c}\right)$ to $\left(L^{0}(\mathcal{F}, K),|\cdot|_{c}\right)$ (namely previous $\left.E_{c}^{*}\right)$, and $(E, \mathcal{P})_{\epsilon, \lambda}^{*}$ for

the random conjugate space consisting of all continuous module homomorphism from $\left(E, \mathcal{P}_{\epsilon, \lambda}\right)$ to $\left(L^{0}(\mathcal{F}, K),|\cdot|_{\epsilon, \lambda}\right)$ (namely previous $E_{\epsilon, \lambda}^{*}$ ), where $|\cdot|$ denotes the $L^{0}$-norm on $L^{0}(\mathcal{F}, K)$, and $|\cdot|_{c}$ and $|\cdot|_{\epsilon, \lambda}$ denote the locally $L^{0}$-convex topology and $(\epsilon, \lambda)$-topology on $L^{0}(\mathcal{F}, K)$, respectively.

By the way, to contrast with the results obtained in [41], we will mention some results of [41] in some places of this section in time.

\subsection{Random compatible topology}

Definition 6.1.1 [29]. Let $E$ and $F$ be two left modules over the algebra $L^{0}(\mathcal{F}, K)$ and the mapping $\langle\cdot, \cdot\rangle: E \times F \rightarrow L^{0}(\mathcal{F}, K)$ a bi-module homomorphism. Then $E$ and $F$ are called a pair in random duality with respect to $\langle\cdot, \cdot\rangle$ over $K$ with base $(\Omega, \mathcal{F}, P)$ if the following axioms are satisfied:

(1) $\langle x, y\rangle=0$ for each $y \in F$ iff $x=0$;

(2) $\langle x, y\rangle=0$ for each $x \in E$ iff $y=0$.

For the sake of convenience,we also say that $\langle E, F\rangle$ is a random duality pair over $K$ with base $(\Omega, \mathcal{F}, P)$ if $E, F$ and $\langle\cdot, \cdot\rangle$ satisfy the above two conditions.

One can easily find that the notion of the left (right) regularity of a random duality pair $\langle E, F\rangle$ as introduced in [41] is equivalent to saying that $E$ (resp. $F$ ) has the countable concatenation property.

Given a random duality pair $\langle E, F\rangle$ over $K$ with base $(\Omega, \mathcal{F}, P), \sigma(E, F)$ always denotes the family $\left\{\|\cdot\|_{f}: f \in F\right\}$ of $L^{0}$-seminorms on $E$, where $\|\cdot\|_{f}: E \rightarrow L_{+}^{0}(\mathcal{F})$ is defined by $\|e\|_{f}=|\langle e, f\rangle|, \forall e \in$ E.

To establish the representation theorem of the random conjugate space $(E, \sigma(E, F))_{c}^{*}$, let us first recall a piece of linear functionals (see [2, Theorem 21.17]): let $E$ be a linear space over $K$, $f_{1}, f_{2}, \ldots, f_{n}$ and $g$ linear functionals on $E$, then there are $\alpha_{1}, \alpha_{2}, \ldots, \alpha_{n}$ in $K$ such that $g=$ $\sum_{k=1}^{n} \alpha_{k} f_{k}$ iff $\bigcap_{k=1}^{n} N\left(f_{k}\right) \subset N(g)$, where $N(f)$ stands for the null space of a linear functional $f$ on $E$. When we generalized the classical result to $L^{0}$-linear functions on an $L^{0}(\mathcal{F}, K)$-module $E$ in [29], we again made use of the countable concatenation property of $L^{0}(\mathcal{F}, K)$, and thus this generalization is not trivial, as shown in [41].

Proposition 6.1.1 [29, 41]. Let $E$ be a left module over the algebra $L^{0}(\mathcal{F}, K), f_{1}, f_{2}, \ldots, f_{n}$ and $g L^{0}$-linear functions on $E$. Then there exist $\xi_{1}, \xi_{2}, \ldots, \xi_{n}$ in $L^{0}(\mathcal{F}, K)$ such that $g=\sum_{k=1}^{n} \xi_{k} f_{k}$ iff $\bigcap_{k=1}^{n} N\left(f_{k}\right) \subset N(g)$.

Proposition 6.1 .2 below was first obtained in [29] because the very random conjugate space $E_{c}^{*}$ was employed in [29].

Proposition 6.1.2 [29]. Let $\langle E, F\rangle$ be a random duality pair over $K$ with base $(\Omega, \mathcal{F}, \mathcal{P})$. Then $(E, \sigma(E, F))_{c}^{*}=F$, namely there exists a unique $y$ in $F$ for each $f \in(E, \sigma(E, F))_{c}^{*}$ such that $f(x)=\langle x, y\rangle, \forall x \in E$.

Remark 6.1.1. In [41], we proved that for each $f \in(E, \sigma(E, F))_{\epsilon, \lambda}^{*}$ there exist a countable subset $\left\{y_{n} \mid n \in N\right\}$ in $F$ and a countable partition $\left\{A_{n} \mid n \in N\right\}$ of $\Omega$ to $\mathcal{F}$ such that $f(x)=$ $\sum_{n \geqslant 1} \tilde{I}_{A_{n}}\left\langle x, y_{n}\right\rangle, \forall x \in E$. 
Definition 6.1.2. $\quad$ Let $\langle E, F\rangle$ be a random duality pair over $K$ with base $(\Omega, \mathcal{F}, \mathcal{P})$. A Hausdorff locally $L^{0}$-convex topology $\mathcal{T}$ for $E$ (namely $(E, \mathcal{T})$ forms a Hausdorff locally $L^{0}$-convex module) is called a random compatible topology with $\langle E, F\rangle$ if $E_{c}^{*}=F$.

Remark 6.1.2. In Definition 6.1.2, $E_{c}^{*}$ is exactly the $L^{0}$-module of continuous module homomorphisms from $(E, \mathcal{T})$ to $\left(L^{0}(\mathcal{F}, K),|\cdot|_{c}\right)$. In [41], we say that a family $\mathcal{P}$ of $L^{0}$-seminorms on $E$ is a random compatible structure with $\langle E, F\rangle$ if $F$ has the countable concatenation property, $(E, \mathcal{P})$ is a random locally convex module and $(E, \mathcal{P})_{\epsilon, \lambda}^{*}=F$.

Proposition 6.1.3 (Mackey topology). Let $\langle E, F\rangle$ be a random duality pair. Then there is the greatest random compatible topology for $E$ with $\langle E, F\rangle$, called random Mackey topology.

Definition 6.1.3 [41]. Let $\langle E, F\rangle$ be a random duality pair, $A \subset E$ and $B \subset F . A^{0}=\{y \in$ $F||\langle x, y\rangle \mid \leqslant 1, \forall x \in A\}$ is called the polar of $A$, and $B^{0}=\{x \in E||\langle x, y\rangle \mid \leqslant 1, \forall y \in B\}$ is called the polar of $B$.

Definition 6.1.4. $\quad$ Let $(E, \mathcal{T})$ be a locally $L^{0}$-convex module over $K$ with base $(\Omega, \mathcal{F}, P)$ and $A \subset E$. Then $A$ is $\mathcal{T}$-bounded if $A$ can be $L^{0}$-absorbed by every neighborhood $U$ of $0 \in E$ (namely there exists some $\xi \in L_{++}^{0}(\mathcal{F})$ such that $\left.A \subset \xi U\right)$.

Proposition 6.1.4. $\quad$ Let $(E, \mathcal{T})$ be a locally $L^{0}$-convex module over $K$ with base $(\Omega, \mathcal{F}, P)$ and $A \subset E$. Then $A$ is $\mathcal{T}$-bounded iff $\vee\{\|a\| \mid a \in A\} \in L_{+}^{0}(\mathcal{F})$ for every $\|\cdot\| \in \mathcal{P}$, where $\mathcal{P}$ is a family of $L^{0}$-seminorms on $E$ which generates $\mathcal{T}$.

Given a locally $L^{0}$-convex module $(E, \mathcal{T})$ over $K$ with base $(\Omega, \mathcal{F}, P), E_{c}^{*}$ denotes the random conjugate space of $(E, \mathcal{T})$, namely $E_{c}^{*}$ denotes the $L^{0}$-module of continuous module homomorphisms from $(E, \mathcal{T})$ to $\left(L^{0}(\mathcal{F}, K),|\cdot|_{c}\right)$. Then $\left\langle E, E_{c}^{*}\right\rangle$ forms a random duality pair over $K$ with base $(\Omega, \mathcal{F}, P)$ with $\langle\cdot, \cdot\rangle: E \times E_{c}^{*} \rightarrow L^{0}(\mathcal{F}, K)$ defined by $\langle g, f\rangle=f(g), \forall(g, f) \in E \times E_{c}^{*}$. As usual, we briefly use $\sigma_{c}\left(E, E^{*}\right)$ for $\sigma_{c}\left(E, E_{c}^{*}\right)$.

Proposition 6.1.4 shows that the notion of a $\mathcal{T}$-bounded set is equivalent to that of an a.s. bounded set in terms of [28], and thus a result of [28] has implied the following:

Proposition 6.1.5 [28]. Let $(E, \mathcal{T})$ be a Hausdorff locally $L^{0}$-convex module over $K$ with base $(\Omega, \mathcal{F}, P)$ and $A \subset E$. Then $A$ is $\mathcal{T}$-bounded iff $A$ is $\sigma_{c}\left(E, E^{*}\right)$-bounded, namely $f(A)$ is $|\cdot|_{c}$-bounded in $\left(L^{0}(\mathcal{F}, K),|\cdot|_{c}\right)$ for each $f \in E_{c}^{*}$.

Definition 6.1.5. $\quad$ Let $(E, \mathcal{T})$ be a Hausdorff locally $L^{0}$-convex module such that $S$ has the countable concatenation property. For a subset $A$ of $E$, then the set $\mathcal{T}-B C_{4}(A)=\cap\{G \subset E \mid G \supset A$ and $G$ is an $L^{0}$-balanced, $L^{0}$-convex and $\mathcal{T}$-closed set with the countable concatenation property $\}$ is called the $L^{0}$-balanced, $L^{0}$-convex, $\mathcal{T}$-closed countable concatenation hull of $A$.

Proposition 6.1.6 (Random bipolar theorem). $\quad$ Let $\langle E, F\rangle$ be a random duality pair such that $E$ has the countable concatenation property. Then $\mathcal{T}-B C_{4}(A)=A^{00}$ for each subset $A$ of $E$ and for each random compatible topology of $\mathcal{T}$.

Remark 6.1.3. The reason why random bipolar theorem is so complicated is that its proof needs the use of the hyperplane separation theorem under the locally $L^{0}$-convex topology - Proposition 5.2 , so that the reader can easily see why we need to consider the countable concatenation operations twice. Random bipolar theorem under the $(\epsilon, \lambda)$-topology is closer to the classical bipolar theorem, see [41, Theorem 3.4]. 


\subsection{Random admissible topology}

Definition 6.2.1. Let $\langle E, F\rangle$ be a random duality pair over $K$ with base $(\Omega, \mathcal{F}, P)$ and $\mathcal{A}$ a family of $\sigma_{c}(F, E)$-bounded subsets of $F$. For each $A \in \mathcal{A}$, let $\|x\|_{A}=\vee\{|\langle x, y\rangle| \mid y \in A\}, \forall x \in E$ $\left(\|\cdot\|_{A}\right.$ is well defined by Proposition 6.1.4). Then the locally $L^{0}$-convex topology induced by the family $\mathcal{P}:=\left\{\|\cdot\|_{A}: A \in \mathcal{A}\right\}$ of $L^{0}$-seminorms on $E$, denoted by $\mathcal{T}_{\mathcal{A}}$, is called the random uniform convergence topology of $E$ over $\mathcal{A}$. Furthermore, if $\left(E, \mathcal{T}_{\mathcal{A}}\right)_{c}^{*} \supset F$ then $\mathcal{T}_{\mathcal{A}}$ is called a random admissible topology of $E$ with respect to $\langle E, F\rangle$.

Proposition 6.2.1. Let $\langle E, F\rangle, \mathcal{A}$ and $\mathcal{T}_{\mathcal{A}}$ be the same as in Definition 6.2.1. Then $\mathcal{T}_{\mathcal{A}}$ is Hausdorff iff $\cup \mathcal{A}$ is total for $E$, namely $\langle x, y\rangle=0 \forall y \in \cup \mathcal{A}$ implies $x=\theta$, in turn iff $\operatorname{span}(\mathcal{A}):=$ the submodule generated by $\cup \mathcal{A}$, is $\sigma_{\epsilon, \lambda}(F, E)$-dense in $F$.

Definition 6.2.2 [15]. $\quad$ Let $(E, \mathcal{T})$ be a locally $L^{0}$-convex module over $K$ with base $(\Omega, \mathcal{F}, P)$. An $L^{0}$-seminorm $\|\cdot\|: E \rightarrow L_{+}^{0}(\mathcal{F})$ is called $\mathcal{T}$-lower semicontinuous if for each $\xi \in L_{+}^{0}(\mathcal{F})$ the set $\{x \in E \mid\|x\| \leqslant \xi\}$ is $\mathcal{T}$-closed.

Proposition 6.2.2. Let $\langle E, F\rangle$ be a random duality pair such that E has the countable concatenation property. Then a locally $L^{0}$-convex topology $\mathcal{T}$ for $E$ is a random admissible topology iff $\mathcal{T}$ satisfies the following two conditions:

(1) $\mathcal{T} \supset \sigma_{c}(E, F)$;

(2) $\mathcal{T}$ is induced by a family of $\sigma_{c}(E, F)$-lower semicontinuous $L^{0}$-seminorms on $E$.

Besides, the above (2) is equivalent to the following:

(3) There is a neighborhood base $\mathcal{U}$ of $0 \in E$ for $\mathcal{T}$ such that each $U \in \mathcal{U}$ is an $L^{0}$-convex, $L^{0}$-balanced, $L^{0}$-absorbent and $\sigma_{c}(E, F)$-closed set with the countable concatenation property.

Proposition 6.2.3. $\quad$ Let $(E, \mathcal{T})$ be a locally $L^{0}$-convex module over $K$ with base $(\Omega, \mathcal{F}, P)$ such that $E$ has the countable concatenation property. Suppose $\mathcal{E}$ is the family of $\mathcal{T}$-equicontinuous subsets of $E_{c}^{*}$, then $\mathcal{T}=\mathcal{T}_{\mathcal{E}}$, and hence $\mathcal{T}$ is a random admissible topology of $E$ with respect to the natural pair $\left\langle E, E_{c}^{*}\right\rangle$, where we say that a subset $H$ of $E_{c}^{*}$ is $\mathcal{T}$-equicontinuous if $H$ is an equicontinuous family of mappings from $(E, \mathcal{T})$ to $\left(L^{0}(\mathcal{F}, K),|\cdot|_{c}\right)$.

Definition 6.2.3. Let $\langle E, F\rangle$ be a random duality pair over $K$ with base $(\Omega, \mathcal{F}, P)$ such that $F$ has the countable concatenation property. A family $\mathcal{B}$ of $\sigma_{c}(F, E)$-bounded subsets of $F$ is called saturated if the following are satisfied:

(1) If $B \in \mathcal{B}$ and $A \subset B$, then $A \in \mathcal{B}$;

(2) If $A, B \in \mathcal{B}$, then $A \cup B \in \mathcal{B}$;

(3) If $B \in \mathcal{B}$, then $\sigma_{c}(F, E)-B C_{4}(B) \in \mathcal{B}$ (see Definition 6.1 .5 for the operation $\left.B C_{4}\right)$;

(4) If $B \in \mathcal{B}$ and $\lambda \in L^{0}(\mathcal{F}, K)$, then $\lambda B \in \mathcal{B}$.

Proposition 6.2.4. Let $\langle E, F\rangle$ be a random duality pair such that $F$ has the countable concatenation property. If $\mathcal{B}$ is a saturated family of $\sigma_{c}(F, E)$-bounded subsets of $F$, then $\mathcal{T}_{\mathcal{B}}$ is random admissible iff $\cup \mathcal{B}=F$.

Definition 6.2.4 [15]. Let $(E, \mathcal{T})$ be a locally $L^{0}$-convex module. A subset $G$ of $E$ is called an $L^{0}$-barrel if it is $L^{0}$-convex, $L^{0}$-absorbent, $L^{0}$-balanced and $\mathcal{T}$-closed. If every $L^{0}$-barrel is a neighborhood of $0 \in E$, then $(E, \mathcal{T})$ is called an $L^{0}$-barreled module. 
Definition 6.2.5. $\quad$ Let $(E, \mathcal{T})$ be a locally $L^{0}$-convex module. If every $L^{0}$-barrel with the countable concatenation property is a neighborhood of $0 \in E$, then $(E, \mathcal{T})$ is called an $L^{0}$-pre-barreled module.

Clearly, the two notions of an $L^{0}$-barreled module and an $L^{0}$-pre-barreled module coincide for an ordinary locally convex space, but the latter is weaker than the former in general. Up to now, we have not yet known what kind of locally $L^{0}$-convex module is $L^{0}$-barreled. Fortunately, we have the following:

Proposition 6.2.5. Let $(E, \mathcal{T})$ be a locally $L^{0}$-convex module such that $E$ has the countable concatenation property. Then $E$ is an $L^{0}$-pre-barreled module iff $\mathcal{T}=\beta\left(E, E_{c}^{*}\right)$, where $\beta\left(E, E_{c}^{*}\right)$ is the random uniform convergence topology of $E$ over the family of all $\sigma_{c}\left(E^{*}, E\right)$-bounded subsets of $E_{c}^{*}$.

Proposition 6.2.6 below is the most important result in this section, since it is enough to meet the current needs of the theory of conditional risk measures.

Proposition 6.2.6. $\quad$ Every complete $R N$ module $(E,\|\cdot\|)$ such that $E$ has the countable concatenation property is an $L^{0}$-pre-barreled module when it is endowed with the locally $L^{0}$-convex topology. Specially, $L_{\mathcal{F}}^{p}(\mathcal{E})$ is an $L^{0}$-pre-barreled module.

\section{$7 \quad L^{0}$-convex analysis and its applications to conditional risk measures}

The purpose of this section is to generalize the following three basic theorems in classical convex analysis to random metric theory and to apply the generalized basic theorems to the theory of conditional risk measures.

To introduce the three basic theorems, let $E$ be a real Hausdorff locally convex space and $E^{\prime}$ the classical conjugate space of $E$. An extended real-valued convex function $f: E \rightarrow[-\infty,+\infty]$ is called proper if $f(x)>-\infty$ for all $x \in E$ and $\operatorname{dom}(f)=\{x \in E \mid f(x)<+\infty\} \neq \emptyset$. Besides, $\operatorname{int}(\operatorname{dom}(f))$ denotes the interior of $\operatorname{dom}(f)$.

Theorem A [14]. Every proper extended real-valued lower semicontinuous convex function $f$ defined on a barreled space $E$ is continuous on $\operatorname{int}(\operatorname{dom}(f))$.

Theorem B [14]. Every proper extended real-valued lower semicontinuous convex function $f$ defined on a barreled space $E$ is subdifferentiable on $\operatorname{int}(\operatorname{dom}(f))$.

Theorem C [14]. For every proper extended real-valued lower semicontinuous convex function $f$ defined on any locally convex space $E, f^{* *}=f$, where $f^{* *}: E \rightarrow[-\infty,+\infty]$ is defined by $f^{* *}(x)=\sup \left\{u(x)-f^{*}(u) \mid u \in E^{\prime}\right\}, \forall x \in E$ and $f^{*}: E^{\prime} \rightarrow[-\infty,+\infty]$ is defined by $f^{*}(u)=$ $\sup \{u(x)-f(x) \mid x \in E\}, \forall u \in E^{\prime}$.

The so-called $L^{0}$-convex analysis is convex analysis of the $L^{0}$-valued $L^{0}$-convex functions defined on random locally convex modules. $L^{0}$-convex analysis and its applications to conditional risk measures were first studied by Filipović et al. in [15], which is, without doubt, an excellent contribution to both random metric theory and the theory of conditional risk measures. On the other hand, there were some negligences in their paper [15], their main results will be improved based on Proposition 5.3 in the process of presenting their results. Besides, to pave the way for applying random metric theory to conditional risk measures, we further give the new continuity and subdifferentiability the- 
orems for $L^{0}$-convex functions defined on $L^{0}$-pre-barreled modules because it is not very convenient for the corresponding theorems given in [15] to be applied to conditional risk measures. In particular, we also give a pleasant $(\varepsilon, \lambda)$-topological version of Fenchel-Moreau type dual representation theorem for $L^{0}$-convex functions, which contains the corresponding locally $L^{0}$-convex topological version of Fenchel-Moreau type dual representation theorem established in [15] as a special case.

By the way, in this section the results without mention of a reference belong to the author. To simplify the notation, let $(\Omega, \mathcal{E}, P)$ be a fixed probability space, $\mathcal{F}$ a fixed sub $\sigma$-algebra of $\mathcal{E}$, we make the following convention: $L^{0}(\mathcal{E}):=L^{0}(\mathcal{E}, R), \bar{L}^{0}(\mathcal{E}):=\bar{L}^{0}(\mathcal{E}, R), L^{p}(\mathcal{E}):=\{\xi \in$ $\left.\left.L^{0}(\mathcal{E}, R)\left|\int_{\Omega}\right| \xi\right|^{p} d P<+\infty\right\}$ for $1 \leqslant p<+\infty$ and $L^{\infty}(\mathcal{E}):=\left\{\xi \in L^{0}(\mathcal{E}, R) \mid \xi\right.$ is essentially bounded\}.

Similarly, one can easily understand the notions such as $L^{0}(\mathcal{F}), \bar{L}^{0}(\mathcal{F})$ and $L^{p}(\mathcal{F})(1 \leqslant p \leqslant+\infty)$.

\section{$7.1 \quad L^{0}$-convex functions}

Let $E$ be a left module over the algebra $L^{0}(\mathcal{F})$. The effective domain of a function $f: E \rightarrow \bar{L}^{0}(\mathcal{F})$ is denoted by $\operatorname{dom}(f):=\left\{x \in E \mid f(x) \in L^{0}(\mathcal{F})\right\}$. The epigraph of $f$ is denoted by epi $(f):=$ $\left\{(x, y) \in E \times L^{0}(\mathcal{F}) \mid f(x) \leqslant y\right\}$. The function $f$ is called proper if $f(x)>-\infty$ on $\Omega$ for every $x \in E$ and $\operatorname{dom}(f) \neq \emptyset$.

Definition 7.1.1 $[15,16]$. Let $E$ be a left module over the algebra $L^{0}(\mathcal{F})$ and $f: E \rightarrow \bar{L}^{0}(\mathcal{F})$.

(1) $f$ is $L^{0}(\mathcal{F})$-convex if $f(\xi x+(1-\xi) y) \leqslant \xi f(x)+(1-\xi) f(y)$ for all $x$ and $y$ in $E$ and $\xi \in L_{+}^{0}(\mathcal{F})$ such that $0 \leqslant \xi \leqslant 1$ (Here we make the convention that $0 \cdot( \pm \infty)=0$ and $\infty-\infty=\infty$ ).

(2) $f$ has the local property if $\tilde{I}_{A} f(x)=\tilde{I}_{A} f\left(\tilde{I}_{A} x\right)$ for all $x \in E$ and $A \in \mathcal{F}$.

(3) $f$ is regular if $\tilde{I}_{A} f(x)=f\left(\tilde{I}_{A} x\right)$ for all $x \in E$ and $A \in \mathcal{F}$.

Proposition 7.1.1 $[15,16] . \quad f: E \rightarrow \bar{L}^{0}(\mathcal{F})$ is $L^{0}(\mathcal{F})$-convex iff $f$ has the local property and epi(f) is $L^{0}(\mathcal{F})$-convex.

Definition 7.1.2. $\quad$ Let $(E, \mathcal{F})$ be a random locally convex module over $K$ with base $(\Omega, \mathcal{F}, P)$. A function $f: E \rightarrow L^{0}(\mathcal{F})$ is called $\mathcal{T}_{\epsilon, \lambda}$-continuous if it is continuous from $\left(E, \mathcal{T}_{\epsilon, \lambda}\right)$ to $\left(L^{0}(\mathcal{F}, K), \mathcal{T}_{\epsilon, \lambda}\right)$. A function $f: E \rightarrow L^{0}(\mathcal{F}, K)$ is called $\mathcal{T}_{c}$-continuous if it is continuous from $\left(E, \mathcal{T}_{c}\right)$ to $\left(L^{0}(\mathcal{F}, K), \mathcal{T}_{c}\right)$.

When is $L^{0}(\mathcal{F})$-convex a function : $E \rightarrow L^{0}(\mathcal{F})$ if it is a convex (in the usual sense) function defined on an $L^{0}(\mathcal{F})$-module? We have the following pleasant results:

Proposition 7.1.2. Let $(E, \mathcal{P})$ be a random locally convex module over $R$ with base $(\Omega, \mathcal{F}, P)$. Then a $\mathcal{T}_{\epsilon, \lambda}$-continuous function $f: E \rightarrow L^{0}(\mathcal{F})$ is $L^{0}(\mathcal{F})$-convex iff $f$ is convex and has the local property.

Similarly, we can also obtain the following:

Proposition 7.1.3. Let $f$ be a continuous function from $L^{p}(\mathcal{E})$ to $L^{r}(\mathcal{F})(1 \leqslant p, r \leqslant+\infty)$. Then $f$ is $L^{0}(\mathcal{F})$-convex (namely $f(\xi x+(1-\xi) y) \leqslant \xi f(x)+(1-\xi) f(y)$, for all $x, y \in L^{p}(\mathcal{E})$ and $\xi \in L_{+}^{0}(\mathcal{F})$ such that $0 \leqslant \xi \leqslant 1$ ) iff $f$ is convex and has the local property (namely $\tilde{I}_{A} f(x)=\tilde{I}_{A} f\left(\tilde{I}_{A} x\right)$ for all $x \in L^{p}(\mathcal{E})$ and $\left.A \in \mathcal{F}\right)$. 
Recent progress in random metric theory and its applications to conditional risk measures

\subsection{Lower semicontinuity}

Definition 7.2.1. $\quad$ Let $(E, \mathcal{P})$ be a random locally convex module over $R$ with base $(\Omega, \mathcal{F}, P)$. A function $f: E \rightarrow \bar{L}^{0}(\mathcal{F})$ is called $\mathcal{T}_{\epsilon, \lambda}$-lower semicontinuous if epi(f) is closed in $\left(E, \mathcal{T}_{\epsilon, \lambda}\right) \times$ $\left(L^{0}(\mathcal{F}), \mathcal{T}_{\epsilon, \lambda}\right)$. A function $f: E \rightarrow \bar{L}^{0}(\mathcal{F})$ is called $\mathcal{T}_{c}$-lower semicontinuous if epi(f) is closed in $\left(E, \mathcal{T}_{c}\right) \times\left(L^{0}(\mathcal{F}), \mathcal{T}_{c}\right)$

Proposition 7.2.1. $\quad$ Let $(E, \mathcal{P})$ be a random locally convex module over $R$ with base $(\Omega, \mathcal{F}, P)$ such that both $E$ and $\mathcal{P}$ have the countable concatenation property. If $f: E \rightarrow \bar{L}^{0}(\mathcal{F})$ is a function with the local property. Then the following are equivalent to each other:

(1) $f$ is $\mathcal{T}_{c}$-lower semicontinuous;

(2) $\{x \in E \mid f(x) \leqslant r\}$ is $\mathcal{T}_{c}$-closed for each $r \in L^{0}(\mathcal{F})$;

(3) $\varliminf_{\alpha} f\left(x_{\alpha}\right) \geqslant f\left(x_{0}\right)$ for each $x_{0} \in E$ and each net $\left\{x_{\alpha}, x \in \Gamma\right\}$ in $E$ such that $\left\{x_{\alpha}, \alpha \in \Gamma\right\}$ is $\mathcal{T}_{c}$-convergent to $x_{0}$. Where $\underline{\lim }_{\alpha} f\left(x_{\alpha}\right)=\vee_{\alpha \in \Gamma}\left(\wedge_{\beta} \geqslant \alpha f\left(x_{\beta}\right)\right)$.

Remark 7.2.1. Proposition 7.2.1 first occurred in [15, Proposition 3.4 and Lemma 3.10] where the countable concatenation property of $E$ was not assumed, but this condition should be added since Lemma 2.28 of [15] has been improved to Proposition 5.3.

For the $(\epsilon, \lambda)$-topology, we only have the following:

Proposition 7.2.2. Let $(E, \mathcal{P})$ be a random locally convex module over $R$ with base $(\Omega, \mathcal{F}, P)$ and $f: E \rightarrow \bar{L}^{0}(\mathcal{F})$ a function. Then we have the following statements:

(1) $f$ is $\mathcal{T}_{\epsilon, \lambda}$-lower semicontinuous if ${\underline{\varliminf_{\alpha}}}_{\alpha} f\left(x_{\alpha}\right) \geqslant f\left(x_{0}\right)$ for each $x_{0} \in E$ and each net $\left\{x_{\alpha}, \alpha \in \Gamma\right\}$ in $E$ such that $\left\{x_{\alpha}, \alpha \in \Gamma\right\}$ is $\mathcal{T}_{\epsilon, \lambda}$-convergent to $x_{0}$;

(2) $\{x \in E \mid f(x) \leqslant r\}$ is $\mathcal{T}_{\epsilon, \lambda}$-closed for each $r \in L^{0}(\mathcal{F})$ if $f$ is $\mathcal{T}_{\epsilon, \lambda}$ - lower semicontinuous.

In general, a $\mathcal{T}_{\epsilon, \lambda}$-lower semicontinuous function must be $\mathcal{T}_{c}$-lower semicontinuous. On the other hand, Proposition 2.2.5 leads to the following nice result:

Proposition 7.2.3. Let $(E, \mathcal{P})$ be a random locally convex module over $R$ with base $(\Omega, \mathcal{F}, P)$ such that $E$ has the countable concatenation property and $f: E \rightarrow \bar{L}^{0}(\mathcal{F})$ a function with the local property. Then $f$ is $\mathcal{T}_{\epsilon, \lambda}$-lower semiconinuous iff $f$ is $\mathcal{T}_{c}$-lower semicontinuous, specially this is true for an $L^{0}(\mathcal{F})$-convex function $f$.

\subsection{Continuity and subdifferentiability}

Let $(E, \mathcal{T})$ be a locally $L^{0}$-convex module over $R$ with base $(\Omega, \mathcal{F}, P)$ and $f: E \rightarrow \bar{L}^{0}(\mathcal{F})$ a proper $\mathcal{T}$-lower semicontinuous $L^{0}(\mathcal{F})$-convex function. In this section, $\operatorname{int}(\operatorname{dom}(f))$ denotes the $\mathcal{T}$-interior of $\operatorname{dom}(f)$. Further, $u \in E_{c}^{*}$ is called a subgradient of $f$ at $x_{0} \in \operatorname{dom}(f)$ if $u\left(x-x_{0}\right) \leqslant$ $f(x)-f\left(x_{0}\right), \forall x \in E$, and $\partial f\left(x_{0}\right)$ denotes the set of subgradients of $f$ at $x_{0}$. If $\partial f\left(x_{0}\right) \neq \emptyset$, then $f$ is called $\mathcal{T}$-subdifferentiable.

Proposition 7.3.1 [15]. $\quad$ Let $(E, \mathcal{T})$ be an $L^{0}$-barreled module over $R$ with base $(\Omega, \mathcal{F}, P)$ and $f: E \rightarrow \bar{L}^{0}(\mathcal{F})$ a proper $\mathcal{T}$-lower semicontinuous $L^{0}$-convex function. Then $f$ is $\mathcal{T}$-continuous on $\operatorname{int}(\operatorname{dom}(f))$.

Proposition 7.3.2 [15]. $\quad$ Let $(E, \mathcal{T})$ and $f$ be the same as in Proposition 7.3.1. Then $\partial f\left(x_{0}\right) \neq \emptyset$ for all $x_{0} \in \operatorname{int}(\operatorname{dom}(f))$. 
Remark 7.3.1. Proposition 7.3.2 is just Theorem 3.7 of [15] where $(E, \mathcal{T})$ was assumed to have the countable concatenation property in the sense of [15] (namely, $\mathcal{T}$ can be a family $\mathcal{P}$ of $L^{0}$ seminorms on $E$ such that $\mathcal{P}$ has the countable concatenation property), but the assumption was not really used in the proof.

In [15], Filipovic et al. stated in [15, p. 4018] that an $R N$ module would be $L^{0}$-barreled when it is endowed with the locally $L^{0}$-convex topology. But the claim of them is obviously not true, even it is not the case for a classical normed space, either. What is more serious is that up to the present time we have not known if $L_{\mathcal{F}}^{p}(\mathcal{E})$ is an $L^{0}$-barreled module, which considerably reduces the availability of Propositions 7.3.1 and 7.3.2 for conditional risk measures. Fortunately, the two results below resolve the above difficulties!

Proposition 7.3.3. Let $(E, \mathcal{T})$ be an $L^{0}$-pre-barreled module over $R$ with base $(\Omega, \mathcal{F}, P)$ such that $E$ has countable concatenation property and $f: E \rightarrow \bar{L}^{0}(\mathcal{F})$ a proper $\mathcal{T}$-lower semicontinuous $L^{0}$-convex function. Then $f$ is $\mathcal{T}$-continuous on $\operatorname{int}(\operatorname{dom}(f))$.

Proposition 7.3.4. Let $(E, \mathcal{T})$ and $f$ be the same as in Proposition 7.3.3. Then $\partial f\left(x_{0}\right) \neq \emptyset$ for all $x_{0}$ in $\operatorname{int}(\operatorname{dom}(f))$.

\subsection{Fenchel-Moreau type dual representation theorems under the two kinds of topologies}

Let $(E, \mathcal{P})$ be a random locally convex module over $R$ with base $(\Omega, \mathcal{F}, P)$ and $f: E \rightarrow \bar{L}^{0}(\mathcal{F})$ a proper $\mathcal{T}_{\epsilon, \lambda}$-lower semicontinuous $L^{0}$-convex function.

The $\mathcal{T}_{\epsilon, \lambda}$-conjugate function $f_{\epsilon, \lambda}^{*}: E_{\epsilon, \lambda}^{*} \rightarrow \bar{L}^{0}(\mathcal{F})$ of $f$ is defined as follows:

$$
f_{\epsilon, \lambda}^{*}(u)=\vee\{u(x)-f(x) \mid x \in E\}, \quad \forall u \in E_{\epsilon, \lambda}^{*} .
$$

The $\mathcal{T}_{\epsilon, \lambda}$-biconjugate function $f_{\epsilon, \lambda}^{* *}: E \rightarrow \bar{L}^{0}(\mathcal{F})$ of $f$ is defined as follows:

$$
f_{\epsilon, \lambda}^{* *}(x)=\vee\left\{u(x)-f_{\epsilon, \lambda}^{*}(u) \mid u \in E_{\epsilon, \lambda}^{*}\right\}, \quad \forall x \in E .
$$

Then we have the $(\epsilon, \lambda)$-topological version of Fenchel-Moreau type dual representation theorem as follows:

Proposition 7.4.1. $\quad$ Let $(E, \mathcal{P}), f$ and $f_{\epsilon, \lambda}^{* *}$ be the same as above. Then $f_{\epsilon, \lambda}^{* *}=f$.

Let $(E, \mathcal{P})$ be a random locally convex module over $R$ with base $(\Omega, \mathcal{F}, P)$ and $f: E \rightarrow \bar{L}^{0}(\mathcal{F})$ a proper $\mathcal{T}_{c}$-lower semicontinuous $L^{0}$-convex function. Then the $\mathcal{T}_{c}$-conjugate function $f_{c}^{*}: E_{c}^{*} \rightarrow$ $\bar{L}^{0}(\mathcal{F})$ of $f$ is defined by $f_{c}^{*}(u)=\vee\{u(x)-f(x) \mid x \in E\}, \forall u \in E_{c}^{*}$. And the $\mathcal{T}_{c}$-biconjugate function $f_{c}^{* *}: E \rightarrow \bar{L}^{0}(\mathcal{F})$ of $f$ is defined by: $f_{c}^{* *}(x)=\vee\left\{u(x)-f_{c}^{*}(u) \mid u \in E_{c}^{*}\right\}, \forall x \in E$.

Then we can now have the $\mathcal{T}_{c}$-topological version of Fenchel-Moreau type dual representation theorem as follows.

Corollary 7.4.1. $\quad$ Let $(E, \mathcal{P})$ be a random locally convex module over $R$ with base $(\Omega, \mathcal{F}, P)$ such that both $E$ and $\mathcal{P}$ have the countable concatenation property and $f$ a proper $\mathcal{T}_{c}$-lower semicontinuous $L^{0}$-convex function. Then $f_{c}^{* *}=f$.

Remark 7.4.1. Corollary 7.4.1 was first studied in [15] where the countable concatenation property of $E$ was not assumed, but the condition should be added to ensure the feasibility of the proof 
of [15, Theorem 3.8] as given in [15]. Since $E$ has the countable concatenation property, $f$ is proper, $\mathcal{T}_{c}$-lower semicontinuous and $L^{0}(\mathcal{F})$-convex iff $f$ is proper, $\mathcal{T}_{\epsilon, \lambda}$-lower semicontinuous and $L^{0}(\mathcal{F})$ convex by Proposition 7.2.3, and when $\mathcal{P}$ has the countable concatenation property $E_{c}^{*}$ is just $E_{\epsilon, \lambda}^{*}$. Thus Corollary 7.4.1 is a special case of Proposition 7.4.1, and Proposition 7.4.1 seems more natural than Corollary 7.4.1 since Proposition 7.4.1 has the same form as the classical Fenchel-Moreau type dual representation theorem (namely Theorem C).

\subsection{Some applications to conditional risk measures}

Definition 7.5.1 [16]. Let $1 \leqslant p \leqslant+\infty$. A function $f: L_{\mathcal{F}}^{p}(\mathcal{E}) \rightarrow \bar{L}^{0}(\mathcal{F})$ is called:

(1) monotone if $f(x) \leqslant f(y)$ for all $x, y \in L_{\mathcal{F}}^{p}(\mathcal{E})$ such that $x \geqslant y$;

(2) subcash invariant if $f(x+y) \geqslant f(x)-y$ for all $x \in L_{\mathcal{F}}^{p}(\mathcal{E})$ and $y \in L_{+}^{0}(\mathcal{F})$;

(3) cash invariant if $f(x+y)=f(x)-y$ for all $x \in L_{\mathcal{F}}^{p}(\mathcal{E})$ and $y \in L^{0}(\mathcal{F})$;

Further, an $L^{0}(\mathcal{F})$-convex, monotone and cash invariant function from $L_{\mathcal{F}}^{p}(\mathcal{E})$ to $\bar{L}^{0}(\mathcal{F})$ is called an $L^{0}(\mathcal{F})$-convex conditional risk measure.

Remark 7.5.1. An $L^{0}(\mathcal{F})$-convex conditional risk measure in the sense of Definition 7.5.1 is exactly a conditional convex risk measure of $L_{\mathcal{F}}^{p}(\mathcal{E})$-type as mentioned in Section 1.3.

Propositions 7.3.3 and 7.3.4 justify Proposition 7.5.1 below, which was given in [15].

Proposition 7.5.1. A proper $\mathcal{T}_{c}$-lower semicontinuous $L^{0}(\mathcal{F})$-convex function, in particular, a proper $\mathcal{T}_{c}$-lower semicontinuous $L^{0}(\mathcal{F})$-convex conditional risk measure on $L_{\mathcal{F}}^{p}(\mathcal{E})$, is $\mathcal{T}_{c}$-continuous and subdifferentiable on the interior of its effective domain.

Both Proposition 7.4.1 and Corollary 7.4.1 can justify Proposition 7.5.2 below that was first given in [15], but Proposition 7.4.1 seems more convenient for Proposition 7.5.3 below.

Proposition 7.5.2. Let $1 \leqslant p<+\infty$. Every proper $\mathcal{T}_{c}$ (equivalently, $\mathcal{T}_{\epsilon, \lambda}$ )-lower semicontinuous $L^{0}(\mathcal{F})$-convex function $f: L_{\mathcal{F}}^{p}(\mathcal{E}) \rightarrow \bar{L}^{0}(\mathcal{F})$ can be represented as follows:

$$
f(x)=\vee\left\{E(x \cdot y \mid \mathcal{F})-f^{*}(y) \mid y \in L_{\mathcal{F}}^{q}(\mathcal{E})\right\}, \quad \forall x \in L_{\mathcal{F}}^{p}(\mathcal{E}),
$$

where $\frac{1}{p}+\frac{1}{q}=1$ and $f^{*}(y)=\vee\left\{E(x \cdot y \mid \mathcal{F})-f(x) \mid x \in L_{\mathcal{F}}^{p}(\mathcal{E})\right\}, \forall y \in L_{\mathcal{F}}^{q}(\mathcal{E})$.

Proposition 7.5.3. Every proper $\sigma_{\epsilon, \lambda}\left(L_{\mathcal{F}}^{\infty}(\mathcal{E}), L_{\mathcal{F}}^{1}(\mathcal{E})\right.$ ) (equivalently, $\sigma_{c}\left(L_{\mathcal{F}}^{\infty}(\mathcal{E}), L_{\mathcal{F}}^{1}(\mathcal{E})\right)$ )-lower semicontinuous $L^{0}(\mathcal{F})$-convex function $f: L_{\mathcal{F}}^{\infty}(\mathcal{E}) \rightarrow \bar{L}^{0}(\mathcal{F})$ can be represented as follows:

$$
f(x)=\vee\left\{E(x \cdot y \mid \mathcal{F})-f^{*}(y) \mid y \in L_{\mathcal{F}}^{1}(\mathcal{E})\right\}, \quad \forall x \in L_{\mathcal{F}}^{\infty}(\mathcal{E}),
$$

where $f^{*}(y)=\vee\left\{E(x \cdot y \mid \mathcal{F})-f(x) \mid x \in L_{\mathcal{F}}^{\infty}(\mathcal{E})\right\}, \forall y \in L_{\mathcal{F}}^{1}(\mathcal{E})$.

Remark 7.5.2. Proposition 7.5.3 uses the fact that $\left(L_{\mathcal{F}}^{\infty}(\mathcal{E}), \sigma\left(L_{\mathcal{F}}^{\infty}(\mathcal{E}), L_{\mathcal{F}}^{1}(\mathcal{E})\right)\right)_{\epsilon, \lambda}^{*}=L_{\mathcal{F}}^{1}(\mathcal{E})$, which was proved in [41].

When $f$ in Proposition 7.5.2 is a conditional risk measure, the following refined Proposition 7.5.4 can be obtained:

Proposition 7.5.4 [15]. Let $1 \leqslant p<+\infty$. Every proper $\mathcal{T}_{c}$ (equivalently, $\mathcal{T}_{\epsilon, \lambda}$ )-lower semicontinuous $L^{0}(\mathcal{F})$-convex conditional risk measure $f: L_{\mathcal{F}}^{p}(\mathcal{E}) \rightarrow \bar{L}^{0}(\mathcal{F})$ can be represented as follows:

$$
f(x)=\vee\left\{E(x \cdot y \mid \mathcal{F})-f^{*}(y) \mid y \in L_{\mathcal{F}}^{q}(\mathcal{E}), y \leqslant 0 \text { and } E(y \mid \mathcal{F})=-1\right\}, \quad \forall x \in L_{\mathcal{F}}^{p}(\mathcal{E}),
$$


where $\frac{1}{p}+\frac{1}{q}=1$, and $f^{*}(y)$ is understood as in Proposition 7.5.2.

Proposition 7.5.5. When $p=\infty$, Proposition 7.5.4 is also valid if $f$ is a proper $\sigma_{\epsilon, \lambda}\left(L_{\mathcal{F}}^{\infty}(\mathcal{E}), L_{\mathcal{F}}^{1}(\mathcal{E})\right)$ $\left(\right.$ or $\sigma_{c}\left(L_{\mathcal{F}}^{\infty}(\mathcal{E}), L_{\mathcal{F}}^{1}(\mathcal{E})\right)$ )-lower semicontinuous $L^{0}(\mathcal{F})$-convex conditional risk measure on $L_{\mathcal{F}}^{\infty}(\mathcal{E})$.

Example 7.5.1. Let $\gamma>0$ and $1 \leqslant p<+\infty$. Then $\rho_{\gamma}: L_{\mathcal{F}}^{p}(\mathcal{E}) \rightarrow \bar{L}^{0}(\mathcal{F})$ defined by $\rho_{\gamma}(x)=$ $\frac{1}{\gamma} \log E\left(e^{-\gamma x} \mid \mathcal{F}\right), \forall x \in L_{\mathcal{F}}^{p}(\mathcal{E})$, is a proper $\mathcal{T}_{c}$ (also $\mathcal{T}_{\epsilon, \lambda}$ )-lower semicontinuous $L^{0}(\mathcal{F})$-convex conditional risk measure on $L_{\mathcal{F}}^{p}(\mathcal{E})$.

Just as we have pointed out in Section 1.3, only the generalized Fenchel Moreau type dual representation theorems - Proposition 7.4.1 and Corollary 7.4.1, which are founded on the idea of random conjugate spaces, can treat $\rho_{\gamma}$ thoroughly.

\section{Extensions of conditional risk measures}

In this section, we will prove that every conditional convex risk measure of $L^{\infty}$-type which is representable as in Proposition 1.3.1 can be uniquely extended to a proper $\sigma_{\epsilon, \lambda}\left(L_{\mathcal{F}}^{\infty}(\mathcal{E}), L_{\mathcal{F}}^{1}(\mathcal{E})\right.$ )-lower semicontinuous conditional convex risk measure of $L_{\mathcal{F}}^{\infty}(\mathcal{E})$-type so that our Proposition 7.5.5 implies Proposition 1.3.1. What is more important is that we will also prove that every continuous convex conditional risk measure of $L^{p}$-type can be uniquely extended to a $\mathcal{T}_{\epsilon, \lambda}$-continuous conditional convex risk measure from $L_{\mathcal{F}}^{p}(\mathcal{E})$ to $L^{0}(\mathcal{F})$ when $1 \leqslant p<+\infty$ so that Proposition 7.5.4 implies Proposition 1.3.3. Thus the two representation Propositions 7.5.4 and 7.5.5 obtained along the module approach unify all the previous representation Propositions 1.3.3 and 1.3.1 obtained along the vector space approach, respectively.

Lemma 8.1. Let $f: L^{\infty}(\mathcal{E}) \rightarrow L^{\infty}(\mathcal{F})$ be a conditional convex risk measure of $L^{\infty}$-type. Then there exists a unique $\mathcal{T}_{\epsilon, \lambda}$-continuous conditional convex risk measure of $L_{\mathcal{F}}^{\infty}(\mathcal{E})$-type $\bar{f}: L_{\mathcal{F}}^{\infty}(\mathcal{E}) \rightarrow$ $L^{0}(\mathcal{F})$ such that $\left.\bar{f}\right|_{L^{\infty}(\mathcal{E})}=f$.

Proof. Let us first recall the definition of the $L^{0}$-norm $\|\mid \cdot\|_{\infty}: L_{\mathcal{F}}^{\infty}(\mathcal{E}) \rightarrow L_{+}^{0}(\mathcal{F})$ defined by \|\|$x \|_{\infty}=\wedge\left\{\xi \in L_{+}^{0}(\mathcal{F})|\xi \geqslant| x \mid\right\}, \forall x \in L_{\mathcal{F}}^{\infty}(\mathcal{E})$. It is obvious that $\|x \mid\|_{\infty} \in L_{+}^{\infty}(\mathcal{F})$ for any $x \in L^{\infty}(\mathcal{E})$.

Since $x=y+x-y \leqslant y+|x-y| \leqslant y+\mid\|x-y\|_{\infty}$ for any $x$ and $y$ in $L^{\infty}(\mathcal{E})$, we have that $f(x) \geqslant f\left(y+\left\|\left|\|x-y \mid\|_{\infty}\right)=f(y)-\right\| \mid x-y \|_{\infty}\right.$, namely $f(x)-f(y) \geqslant-\|\| x-y\|\|_{\infty}$ and $f(y)-f(x) \leqslant\||| x-y \mid\|_{\infty}$, so that $|f(x)-f(y)| \leqslant\|\mid\| x-y \|_{\infty}$ for all $x$ and $y \in L^{\infty}(\mathcal{E})$. Thus $f$ is uniformly $\mathcal{T}_{\epsilon, \lambda}$-continuous from $\left(L^{\infty}(\mathcal{E}),\left|\|\cdot \mid\|_{\infty}\right)\right.$ to $\left(L^{\infty}(\mathcal{F}),|\cdot|\right)$. Further, since $L^{\infty}(\mathcal{E})$ is $\mathcal{T}_{\epsilon, \lambda}$-dense in $\left(L_{\mathcal{F}}^{\infty}(\mathcal{E}),\|\| \cdot\|\|_{\infty}\right)$ by noticing $L^{\infty}(\mathcal{E})=L^{\infty}\left(L_{\mathcal{F}}^{\infty}(\mathcal{E})\right)$ and making use of Proposition 2.2.4, $f$ has a unique extension $\bar{f}: L_{\mathcal{F}}^{\infty}(\mathcal{E}) \rightarrow L^{0}(\mathcal{F})$ and it is easy to see that $\bar{f}$ is also a conditional convex risk measure of $L_{\mathcal{F}}^{\infty}(\mathcal{E})$-type.

Let $\mathcal{P}=\{Q \mid Q$ is a probability measure on $(\Omega, \mathcal{E})$ such that $Q$ is absolutely continuous with respect to $P\}$ and $\mathcal{P}_{\mathcal{F}}=\{Q \in \mathcal{P} \mid Q=P$ on $\mathcal{F}\}$. Further, we identify any element $Q$ in $\mathcal{P}$ with its Radon-Nikodým derivative $\frac{d Q}{d P} \in L^{1}(\mathcal{E})$, then $\mathcal{P}_{\mathcal{F}}$ can be identified with the set $\{y \in$ $\left.L_{+}^{1}(\mathcal{E}) \mid E(y \mid \mathcal{F})=1\right\}$, still denoted by $\mathcal{P}_{\mathcal{F}}$, where $E(\cdot \mid \mathcal{F})$ denotes the conditional expectation under the probability $P$. Then the random penalty function $\alpha: \mathcal{P}_{\mathcal{F}} \rightarrow \bar{L}^{0}(\mathcal{F})$ in Proposition 1.3.1 can be rewritten as $\alpha(y)=\vee\left\{E(-x y \mid \mathcal{F})-f(x) \mid x \in L^{p}(\mathcal{E})\right\}, \forall y \in \mathcal{P}_{\mathcal{F}}$; and if $f$ satisfies (1) of Proposition 1.3.1 then $f(x)=\vee\left\{E(-x y \mid \mathcal{F})-\alpha(y) \mid y \in \mathcal{P}_{\mathcal{F}}\right\}$. Define $f^{*}:\left\{y \in L^{1}(\mathcal{E}) \mid y \leqslant 0, E(y \mid \mathcal{F})=\right.$ 
$-1\} \rightarrow \bar{L}^{0}(\mathcal{F})$ by $f^{*}(y)=\alpha(-y)$, for any $y \in L^{1}(\mathcal{E})$ such that $y \leqslant 0$ and $E(y \mid \mathcal{F})=-1$, then $f(x)=\vee\left\{E(x y \mid \mathcal{F})-f^{*}(y) \mid y \in L^{1}(\mathcal{E}), y \leqslant 0\right.$ and $\left.E(y \mid \mathcal{F})=-1\right\}$.

Finally, define $\bar{f}^{*}: L_{\mathcal{F}}^{1}(\mathcal{E}) \rightarrow \bar{L}^{0}(\mathcal{F})$ by $\bar{f}^{*}(y)=\vee\left\{E(x y \mid \mathcal{F})-\bar{f}(x) \mid x \in L_{\mathcal{F}}^{\infty}(\mathcal{E})\right\}, \forall y \in L_{\mathcal{F}}^{1}(\mathcal{E})$, where $\bar{f}$ denotes the unique extension of $f$ as obtained in Lemma 8.1. Since $L^{\infty}(\mathcal{E})$ is $\mathcal{T}_{\epsilon, \lambda}$-dense in $L_{\mathcal{F}}^{\infty}(\mathcal{E})$ and $f$ is $\mathcal{T}_{\epsilon, \lambda}$-continuous, it is easy to see that $\bar{f}^{*}(y)=\vee\left\{E(x y \mid \mathcal{F})-f(x) \mid x \in L^{\infty}(\mathcal{E})\right\}, \forall y \in$ $L_{\mathcal{F}}^{1}(\mathcal{E})$, and $\bar{f}^{*}(y)=f^{*}(y)$ for any $y \in L^{1}(\mathcal{E})$ such that $y \leqslant 0$ and $E(y \mid \mathcal{F})=-1$.

Theorem 8.1. Let $f: L^{\infty}(\mathcal{E}) \rightarrow L^{\infty}(\mathcal{F})$ be a conditional convex risk measure of $L^{\infty}$-type. Then the following statements are equivalent to each other:

(1) $f(x)=\vee\left\{E(x y \mid \mathcal{F})-f^{*}(y) \mid y \in L^{1}(\mathcal{E}), y \leqslant 0\right.$ and $\left.E(y \mid \mathcal{F})=-1\right\}, \forall x \in L^{\infty}(\mathcal{E})$;

(2) $f(x)=\vee\left\{E(x y \mid \mathcal{F})-\bar{f}^{*}(y) \mid y \in L_{\mathcal{F}}^{1}(\mathcal{E}), y \leqslant 0\right.$ and $\left.E(y \mid \mathcal{F})=-1\right\}, \forall x \in L^{\infty}(\mathcal{E})$;

(3) $\bar{f}(x)=\vee\left\{E(x y \mid \mathcal{F})-\bar{f}^{*}(y) \mid y \in L_{\mathcal{F}}^{1}(\mathcal{E}), y \leqslant 0\right.$ and $\left.E(y \mid \mathcal{F})=-1\right\}, \forall x \in L_{\mathcal{F}}^{\infty}(\mathcal{E})$;

(4) $\bar{f}$ is a $\sigma_{\epsilon, \lambda}\left(L_{\mathcal{F}}^{\infty}(\mathcal{E}), L_{\mathcal{F}}^{1}(\mathcal{E})\right)$-lower semicontinuous conditional convex risk measure of $L_{\mathcal{F}}^{\infty}(\mathcal{E})$ type from $L_{\mathcal{F}}^{\infty}(\mathcal{E})$ to $L^{0}(\mathcal{F})$.

Proof. $\quad(1) \Rightarrow(2)$ is clear.

$(2) \Rightarrow(1)$. We only need to prove that for each fixed $x$ in $L^{\infty}(\mathcal{E})$ and each fixed $y \in L_{\mathcal{F}}^{1}(\mathcal{E})$ such that $y \leqslant 0$ and $E(y \mid \mathcal{F})=-1, E(x y \mid \mathcal{F})-\bar{f}^{*}(y) \leqslant \vee\left\{E(x \tilde{y} \mid \mathcal{F})-f^{*}(\tilde{y}) \mid \tilde{y} \in L^{1}(\mathcal{E}), \tilde{y} \leqslant 0\right.$ and $E(\tilde{y} \mid \mathcal{F})=-1\}$.

In fact, let $A_{n}=[E(|y| \mid \mathcal{F}) \leqslant n]$ and $y_{n}=I_{A_{n}} \cdot y+\left(1-I_{A_{n}}\right) \cdot(-1)$ for each positive integer $n$, then $y_{n} \in L^{1}(\mathcal{E}), y_{n} \leqslant 0$ and $E\left(y_{n} \mid \mathcal{F}\right)=-1$. Further, since both $E(x z \mid \mathcal{F})$ and $\bar{f}^{*}(z)$ have the local property with respect to $z$ in $L_{\mathcal{F}}^{1}(\mathcal{E})$, then $I_{A_{n}}\left(E(x y \mid \mathcal{F})-\bar{f}^{*}(y)\right)=I_{A_{n}} E\left(x \cdot I_{A_{n}} y \mid \mathcal{F}\right)$ $I_{A_{n}} \cdot \bar{f}^{*}\left(I_{A_{n}} y\right)=I_{A_{n}}\left(E\left(x y_{n} \mid \mathcal{F}\right)-\bar{f}^{*}\left(y_{n}\right)\right) \leqslant I_{A_{n}}\left(\vee\left\{E(x \tilde{y} \mid \mathcal{F})-f^{*}(\tilde{y}) \mid \tilde{y} \in L^{1}(\mathcal{E}), \tilde{y} \leqslant 0\right.\right.$ and $E(\tilde{y} \mid \mathcal{F})=-1\})$. Letting $n \rightarrow \infty$, one can have that $E(x y \mid \mathcal{F})-\bar{f}^{*}(y) \leqslant \vee\left\{E(x \tilde{y} \mid \mathcal{F})-f^{*}(\tilde{y}) \mid \tilde{y} \in\right.$ $L^{1}(\mathcal{E}), \tilde{y} \leqslant 0$ and $\left.E(\tilde{y} \mid \mathcal{F})=-1\right\}$.

$(2) \Rightarrow(3)$. Define $g: L_{\mathcal{F}}^{\infty}(\mathcal{E}) \rightarrow \bar{L}^{0}(\mathcal{F})$ by $g(x)=\vee\left\{E(x y \mid \mathcal{F})-\bar{f}^{*}(y) \mid y \in L_{\mathcal{F}}^{1}(\mathcal{E}), y \leqslant 0\right.$ and $E(y \mid \mathcal{F})=-1)\}, \forall x \in L_{\mathcal{F}}^{\infty}(\mathcal{E})$, then $g$ is $\mathcal{T}_{\epsilon, \lambda}$-lower semicontinuous. From (2) one has that $g(x)=\bar{f}(x), \forall x \in L^{\infty}(\mathcal{E})$, so that $g(x) \geqslant \bar{f}(x), \forall x \in L_{\mathcal{F}}^{\infty}(\mathcal{E})$ since $\bar{f}$ is $\mathcal{T}_{\epsilon, \lambda}$-continuous and $L^{\infty}(\mathcal{E})$ is $\mathcal{T}_{\epsilon, \lambda}$-dense. Eventually, $g(x)=\bar{f}(x), \forall x \in L_{\mathcal{F}}^{\infty}(\mathcal{E})$, since it is obvious that $g(x) \leqslant \bar{f}(x), \forall x \in L_{\mathcal{F}}^{\infty}(\mathcal{E})$.

$(3) \Rightarrow(2)$ is clear.

$(3) \Rightarrow(4)$ is clear.

$(4) \Rightarrow(3)$ is implied by Proposition 7.5.5.

Lemma 8.2. Let $1 \leqslant r \leqslant p<+\infty$ and $f: L^{p}(\mathcal{E}) \rightarrow L^{r}(\mathcal{F})$ be a continuous convex conditional risk measure of $L^{p}$-type. Then $f$ can be uniquely extended to a $\mathcal{T}_{\epsilon, \lambda}$-continuous conditional convex risk measure of $L_{\mathcal{F}}^{p}(\mathcal{E})$-type $\bar{f}$ from $L_{\mathcal{F}}^{p}(\mathcal{E})$ to $L^{0}(\mathcal{F})$.

Proof. We first prove that $f$ is $\mathcal{T}_{\epsilon, \lambda}$-continuous from $\left(L^{p}(\mathcal{E}),|||\cdot| \|_{p}\right)$ to $\left(L^{r}(\mathcal{F}),|\cdot|\right)$, see Section 2.3 for the $L^{0}$-norm $\|\cdot \mid\|_{p}$. To this, we only need to prove that, for each fixed $x_{0} \in L^{p}(\mathcal{E})$ and each sequence $\left\{x_{n}, n \in N\right\}$ in $L^{p}(\mathcal{E})$ such that $\left\{E\left(\left|x_{n}-x_{0}\right|^{p} \mid \mathcal{F}\right), n \in N\right\}$ converges in probability $P$ to 0 , there exists a subsequence $\left\{x_{n_{k}}, k \in N\right\}$ of $\left\{x_{n}, n \in N\right\}$ such that $\left\{f\left(x_{n_{k}}\right), k \in N\right\}$ converges in probability $P$ to $f\left(x_{0}\right)$. Since $f$ is monotone and cash invariant, $f$ must be local, so that we only need to prove that, for any positive number $\delta$, there exists an $\mathcal{F}$-measurable subset $H_{\delta}$ of $\Omega$ and a subsequence $\left\{x_{n_{k}}, k \in N\right\}$ of $\left\{x_{n}, n \in N\right\}$ such that $P\left(\Omega \backslash H_{\delta}\right)>1-\delta$ and $\left\{f\left(x_{n_{k}}\right), k \in N\right\}$ converges in probability $P$ to $f\left(x_{0}\right)$ on $\Omega \backslash H_{\delta}$. In fact, by the Egoroff theorem there are such $H_{\delta}$ 
and $\left\{x_{n_{k}}, k \in N\right\}$ such that $\left\{E\left(\left|x_{n_{k}}-x_{0}\right|^{p} \mid \mathcal{F}\right), k \in N\right\}$ converges uniformly to 0 on $\Omega \backslash H_{\delta}$, so that $\left\{\tilde{I}_{\Omega \backslash H_{\delta}} x_{n_{k}}, k \in N\right\}$ converges to $\tilde{I}_{\Omega \backslash H_{\delta}} x_{0}$ in the usual $L^{p}$-norm $\|\cdot\|_{p}$ by the Lebesgue convergence theorem, hence $\left\{\tilde{I}_{\Omega \backslash H_{\delta}} f\left(x_{n_{k}}\right), k \in N\right\}$ converges in the $L^{r}$-norm to $\tilde{I}_{\Omega \backslash H_{\delta}} f\left(x_{0}\right)$, which implies that $\left\{f\left(x_{n_{k}}\right), k \in N\right\}$ converges in probability $P$ to $f\left(x_{0}\right)$ on $\Omega \backslash H_{\delta}$.

Next, it is easy to observe that $L_{\mathcal{F}}^{p}(\mathcal{E})=\left\{\sum_{n=1}^{\infty} \tilde{I}_{A_{n}} x_{n} \mid\left\{A_{n}, n \in N\right\}\right.$ is a countable partition of $\Omega$ to $\mathcal{F}$ and $\left\{x_{n}, n \in N\right\}$ is a sequence in $\left.L^{p}(\mathcal{E})\right\}$. Though $f$ is not necessarily uniformly $\mathcal{T}_{\epsilon, \lambda}$-continuous, the local property of a conditional convex function motivates us to define $\bar{f}: L_{\mathcal{F}}^{p}(\mathcal{E}) \rightarrow L^{0}(\mathcal{F})$ by

$$
\bar{f}(x)=\sum_{n=1}^{\infty} \tilde{I}_{A_{n}} f\left(x_{n}\right), \forall x=\sum_{n=1}^{\infty} \tilde{I}_{A_{n}} x_{n}
$$

since $f$ is convex, local and continuous, $f$ must be $L^{0}(\mathcal{F})$-convex by Proposition 7.1.3, from which one can see that the definition of $\bar{f}(x)$ is independent of the expression of $x$ and it is not difficult to verify that $\bar{f}$ is a $\mathcal{T}_{\epsilon, \lambda}$-continuous conditional convex risk measure of $L_{\mathcal{F}}^{p}(\mathcal{E})$-type. Finally, Since $L^{p}(\mathcal{E})$ is $\mathcal{T}_{\epsilon, \lambda}$-dense in $L_{\mathcal{F}}^{p}(\mathcal{E})$, an $L_{\mathcal{F}}^{p}(\mathcal{E})$-type of $\mathcal{T}_{\epsilon, \lambda}$-continuous conditional convex risk measure as an extension of $f$ must be unique.

Lemma 8.3. Let $1 \leqslant r \leqslant p<+\infty$ and $u: L^{p}(\mathcal{E}) \rightarrow L^{r}(\mathcal{F})$ be a continuous linear function with the local property. Then $u$ can be uniquely extended to a $\mathcal{T}_{\epsilon, \lambda}$-continuous $L^{0}(\mathcal{F})$-linear function $\bar{u}$ from $L_{\mathcal{F}}^{p}(\mathcal{E})$ to $L^{0}(\mathcal{F})$ such that the $L^{0}$-norm $\|\bar{u}\|$ of $\bar{u}$ satisfies $\|\bar{u}\| \in L^{\frac{p r}{p-r}}(\mathcal{F})$, where $\frac{p r}{p-r}=\infty$ when $p=r$.

Proof. In the proof of Lemma 8.2 only the continuity and the local property of $f$ are used for the existence of a $\mathcal{T}_{\epsilon, \lambda}$-continuous extension $\bar{f}$, thus the same reasoning shows that such a unique $\mathcal{T}_{\epsilon, \lambda}$-continuous linear extension $\bar{u}$ with the local property exists. Since $\bar{u}$ is linear and local, $\bar{u}$ must be regular, namely $\bar{u}\left(\tilde{I}_{A} x\right)=\tilde{I}_{A} \bar{u}(x), \forall x \in L_{\mathcal{F}}^{p}(\mathcal{E})$ and $A \in \mathcal{F}$, hence $\bar{u}$ is also $L^{0}(\mathcal{F})$-linear by the $\mathcal{T}_{\epsilon, \lambda}$-continuity of $\bar{u}$.

By Corollary 3.4, there exists a unique $y \in L_{\mathcal{F}}^{q}(\mathcal{E})$ such that $\bar{u}(x)=E(x y \mid \mathcal{F}), \forall x \in L_{\mathcal{F}}^{p}(\mathcal{E})$, where $q$ is the Hölder conjugate number of $p$. In particular, $u(x)=E(x y \mid \mathcal{F}), \forall x \in L^{p}(\mathcal{E})$, then Proposition 2.5 of $[16]$ shows that $E\left(|y|^{q} \mid \mathcal{F}\right) \in L^{\frac{r(p-1)}{p-r}}(\mathcal{F})$, namely $\|\bar{u}\|=E\left(|y|^{q} \mid \mathcal{F}\right)^{1 / q} \in L^{\frac{p r}{p-r}}(\mathcal{F})$.

Let $f$ and $\bar{f}$ be the same as in Lemma 8.2.

Define $f^{*}:\left\{y \in L^{q}(\mathcal{E}) \mid y \leqslant 0, E\left(|y|^{q} \mid \mathcal{F}\right) \in L^{\frac{r(p-1)}{p-r}}(\mathcal{F})\right.$ and $\left.E(y \mid \mathcal{F})=-1\right\} \rightarrow \bar{L}^{0}(\mathcal{F})$ by $f^{*}(y)=\vee\left\{E(x y \mid \mathcal{F})-f(x) \mid x \in L^{p}(\mathcal{E})\right\}$.

Define $\bar{f}^{*}: L_{\mathcal{F}}^{q}(\mathcal{E}) \rightarrow \bar{L}^{0}(\mathcal{F})$ by $\bar{f}^{*}(y)=\vee\left\{E(x y \mid \mathcal{F})-\bar{f}(x) \mid x \in L_{\mathcal{F}}^{p}(\mathcal{E})\right\}$.

It is easy to see that $\bar{f}^{*}(y)=f^{*}(y), \forall y \in L^{q}(\mathcal{E})$ such that $y \leqslant 0, E\left(|y|^{q} \mid \mathcal{F}\right) \in L^{\frac{r(p-1)}{p-r}}(\mathcal{F})$ and $E(y \mid \mathcal{F})=-1$.

Theorem 8.2. Let $f$ and $\bar{f}$ be the same as in Lemma 8.2. Then the following statements are true and equivalent to each other:

(1) $f(x)=\vee\left\{E(x y \mid \mathcal{F})-f^{*}(y) \mid y \in L^{q}(\mathcal{E}), y \leqslant 0, E\left(|y|^{q} \mid \mathcal{F}\right) \in L^{\frac{r(p-1)}{p-r}}(\mathcal{F})\right.$ and $E(y \mid \mathcal{F})=$ $-1\}, \forall x \in L^{p}(\mathcal{E})$;

(2) $f(x)=\vee\left\{E(x y \mid \mathcal{F})-\bar{f}^{*}(y) \mid y \in L_{\mathcal{F}}^{q}(\mathcal{E}), y \leqslant 0\right.$ and $\left.E(y \mid \mathcal{F})=-1\right\}, \forall x \in L^{p}(\mathcal{E})$;

(3) $\bar{f}(x)=\vee\left\{E(x y \mid \mathcal{F})-\bar{f}^{*}(y) \mid y \in L_{\mathcal{F}}^{q}(\mathcal{E}), y \leqslant 0\right.$ and $\left.E(y \mid \mathcal{F})=-1\right\}, \forall x \in L_{\mathcal{F}}^{p}(\mathcal{E})$.

Proof. (1) is exactly Proposition 1.3 .3 by identifying $y$ in (1) with $E(\cdot y \mid \mathcal{F})$ in Proposition 1.3.3.

$(1) \Rightarrow(2)$ is clear, so that $(2)$ is true. 
$(2) \Rightarrow(3)$ is similar to the proof of $(2) \Rightarrow(3)$ in Theorem 8.1, so that (3) is true (in fact, (3) can be obtained from Proposition 7.5.4).

$(3) \Rightarrow(2)$ is clear.

$(2) \Rightarrow(1)$. Let $y \in L_{\mathcal{F}}^{q}(\mathcal{E})$ be such that $y \leqslant 0$ and $E(y \mid \mathcal{F})=-1$. For each positive integer $n$, let $A_{n}=\left[E\left(|y|^{q} \mid \mathcal{F}\right) \leqslant n\right]$ and $y_{n}=I_{A_{n}} y+\left(1-I_{A_{n}}\right)(-1)$, then $y_{n} \in L^{q}(\mathcal{E}), E\left(\left|y_{n}\right|^{q} \mid \mathcal{F}\right) \in$

$L^{\frac{r(p-1)}{p-r}}(\mathcal{F}), y_{n} \leqslant 0$ and $E\left(y_{n} \mid \mathcal{F}\right)=-1$, then similar to the proof of $(2) \Rightarrow(1)$ of Theorem 8.1 one can complete the remaining part of the proof of $(2) \Rightarrow(1)$.

To draw a conclusion, Lemmas 8.1 and 8.2 show that Definition 7.5.1 unifies both Definitions 1.3.1 and 1.3.2. Further, Theorems 8.1 and 8.2 show that Propositions 7.5.5 and 7.5.4 include Propositions 1.3.1 and 1.3.3 as a special case, respectively. In particular, only the module approach to conditional risk measures based on Definition 7.5.1 can treat thoroughly conditional entropic risk measure $\rho_{\gamma}$ together with many other conditional risk measures as exhibited in [16]. Thus the module approach has striking advantages. It should be expected that the deep development of dynamic risk measures will involve more of random metric theory.

Acknowledgements The author would like to thank Professor Shijian Yan for some invaluable suggestions. When the author finished this paper, he was informed of the three closely related references [64-66] in communication with Professor Jiaan Yan, the author would like to thank Professor Jiaan Yan for providing these references which should have been cited in Section 1.2. This work was supported by National Natural Science Foundation of China (Grant No. 10871016).

\section{References}

[1] Artzner P, Delbaen F, Eber J M, et al. Coherent measures of risk. Math Finance, 1999, 9: $203-228$

[2] Berberian S K. Lectures in Functional Analysis and Operator Theory. New York: SpringerVerlag, 1974

[3] Biagini S, Frittelli M. On continuity properties and dual representation of convex and monotone functionals on Frechet lattices. Working paper, 2006

[4] Bion-Nadal J. Conditional risk measures and robust representation of convex conditional risk measures. CMAP Preprint, 557, 2004

[5] Breckner W W, Scheiber E. A Hahn-Banach extension theorem for linear mappings into ordered modules. Mathematica, 1977, 19: 13-27

[6] Cheridito P, Delbaen F, Eber J M, et al. Coherent multiperiod risk adjusted values and Bellman's principle. Ann Oper Res, 2007, 152: 5-22

[7] Cheridito P, Delbaen F, Kupper M. Dynamic monetary risk measures for bounded discrete-time processes. Electron J Probab, 2006, 11: 57-106

[8] Cheridito P, Li T. Dual characterization of properties of risk measures on Orlicz hearts. Math Financ Econ, 2008, 2: 29-55 
[9] Delbaen F. Coherent risk measures. Cattedra Galileiana, 2000

[10] Delbaen F. Coherent risk measures on general probability spaces. In Advances in Finance and Stochastics, Sandmann K, Schönbucher P J, eds. Berlin: Springer-Verlag, 2002, 1-37

[11] Detlefsen K, Scandolo G. Conditional and dynamic convex risk measures. Finance Stochast, 2005, 9: 539-561

[12] Diestel J, Uhl Jr J J. Vector Measures, Math Surveys, No. 15. Providence, RI: Amer Math Soc, 1977

[13] Dunford N, Schwartz J T. Linear Operators (I). New York: Interscience, 1957

[14] Ekeland I, Témam R. Convex Analysis and Variational Problems, Chapter (I). Philadelphia: SIAM, 1999

[15] Filipović D, Kupper M, Vogelpoth N. Separation and duality in locally $L^{0}$-convex modules. J Funct Anal, 2009, 256: 3996-4029

[16] Filipović D, Kupper M, Vogelpoth N. Approaches to conditional risk. Working paper Series No. 28, Vienna Institute of Finance, 2009

[17] Filipović D, Svindland G. Convex risk measures beyond bounded risks, or the canonical model space for law-invariant convex risk measures is $L^{1}$. Working Paper Series No. 2, Vienna Institute of Finance, 2008

[18] Föllmer H, Penner I. Convex risk measures and the dynamics of their penalty functions. Statist Decisions, 2006, 24: 61-96

[19] Föllmer H, Schied A. Convex measures of risk and trading constraints. Finance Stochast, 2002, 6: $429-447$

[20] Föllmer H, Schied A. Robust preferences and convex measures of risk. In Advances in Finance and Stochastics, Sandmann K, Schönbucher P J, eds. Berlin: Springer-Verlag, 2002, 39-56

[21] Föllmer H, Schied A. Stochastic Finance, An Introduction in Discrete Time. Berlin-New York: De Gruyter, 2002

[22] Frittelli M, Rosazza Gianin E. Putting order in risk measures. J Bank Finance, 2002, 26: 14731486

[23] Frittelli M, Rosazza Gianin E. Dynamic convex risk measures. In: New Risk Measures for the 21st Century, Szegö G, ed. John Wiley \& Sons, 2004, 227-248

[24] Guo T X. The theory of probabilistic metric spaces with applications to random functional analysis. Master's thesis. Xi'an: Xi'an Jiaotong University, 1989

[25] Guo T X. Random metric theory and its applications. PhD thesis. Xi'an: Xi'an Jiaotong University, 1992 
[26] Guo T X. Extension theorems of continuous random linear operators on random domains. J Math Anal Appl, 1995, 193: 15-27

[27] Guo T X. The Radon-Nikodým property of conjugate spaces and the $\mathrm{w}^{*}$-equivalence theorem for $\mathrm{w}^{*}$-measurable functions. Sci China Ser A, 1996, 39: 1034-1041

[28] Guo T X. Module homomorphisms on random normed modules. Northeast Math J, 1996, 12: $102-114$

[29] Guo T X. Random duality. Xiamen Daxue Xuebao Ziran Kexue Ban, 1997, 36: 167-170

[30] Guo T X. A characterization for a complete random normed module to be random reflexive. Xiamen Daxue Xuebao Ziran Kexue Ban, 1997, 36: 499-502

[31] Guo T X. Some basic theories of random normed linear spaces and random inner product spaces. Acta Anal Funct Appl, 1999, 1: 160-184

[32] Guo T X. Representation theorems of the dual of Lebesgue-Bochner function spaces. Sci China Ser A, 2000, 43: 234-243

[33] Guo T X. Survey of recent developments of random metric theory and its applications in China (I). Acta Anal Funct Appl, 2001, 3: 129-158

[34] Guo T X. Survey of recent developments of random metric theory and its applications in China (II). Acta Anal Funct Appl, 2001, 3: 208-230

[35] Guo T X. The theory of random normed modules and its applications. In: Proceedings of International Conference \& 13th Academic Symposium in China on Functional Space Theory and Its applications, Liu P D, ed. London: Research Information Ltd UK, 2004, 57-66

[36] Guo T X. Several applications of the theory of random conjugate spaces to measurability problems. Sci China Ser A, 2007, 50: 737-747

[37] Guo T X. The relation of Banach-Alaoglu theorem and Banach-Bourbaki-Kakutani-Šmulian theorem in complete random normed modules to stratification structure. Sci China Ser A, 2008, 51: $1651-1663$

[38] Guo T X. A comprehensive connection between the basic results and properties derived from two kinds of topologies for a random locally convex module. arXiv: 0908.1843

[39] Guo T X. Relations between some basic results derived from two kinds of topologies for a random locally convex module. J Funct Anal, 2010, 258: 3024-3047

[40] Guo T X. The theory of module homomorphisms in complete random inner product modules and its applications to Skorohod's random operator theory, to appear

[41] Guo T X, Chen X X. Random duality. Sci China Ser A, 2009, 52: 2084-2098

[42] Guo T X, Li S B. The James theorem in complete random normed modules. J Math Anal Appl, 2005, 308: 257-265 
[43] Guo T X, Peng S L. A characterization for an $L(\mu, K)$-topological module to admit enough canonical module homomorphisms. J Math Anal Appl, 2001, 263: 580-599

[44] Guo T X, Xiao H X. A separation theorem in random normed modules. Xiamen Daxue Xuebao Ziran Kexue Ban, 2003, 42: 270-274

[45] Guo T X, Xiao H X Chen X X. A basic strict separation theorem in random locally convex modules. Nonlinear Anal, 2009, 71: 3794-3804

[46] Guo T X, You Z Y. The Riesz's representation theorem in complete random inner product modules and its applications. Chinese Ann Math Ser A, 1996, 17: 361-364

[47] Guo T X, You Z Y. A note on pointwise best approximation. J Approx Theory, 1998, 93:344-347

[48] Guo T X, Zeng X L. Existence of continuous nontrivial linear functionals on random normed modules. Chinese J Engrg Math, 2008, 25: 117-123

[49] Guo T X, Zeng X L. Random strict convexity and random uniform convexity in random normed modules. Nonlinear Anal, 2010, 73: 1239-1263

[50] Guo T X, Zhao S E, Zeng X L. On analytic foundations of the module approach to conditional risks. to appear

[51] Guo T X, Zhu L H. A characterization of continuous module homomorphisms on random seminormed modules and its applications. Acta Math Sin (Engl Ser), 2003, 19: 201-208

[52] He S W, Wang J G, Yan J A. Semimartingales and Stochastic Analysis. Beijing: Science Press, 1995

[53] Kaina M, Rüschendorf L. On convex risk measures on $L^{p}$. Working paper, 2007

[54] Kantorovic L V. The method of successive approximations for functional equations. Acta Math., 1939, 71: 63-97

[55] Krätschmer V. On $\sigma$-additive robust representation of convex risk measures for unbounded financial positions in the presence of uncertainty about the market model. SFB 649 Discussion Paper 2007-010. Berlin: Humboldt University, 2007

[56] Kupper M, Vogelpoth N. Complete $L^{0}$-normed modules and automatic continuity of monotone convex functions. Working paper Series No.10., Vienna Institute of Finance, 2008

[57] Neveu J. Mathematical Foundations of the Calculus of Probabilities. San Francisco: Holden Day, 1965

[58] Peng S. Nonlinear expectations, nonlinear evaluations and risk measures. In: Lecture notes in Mathematics 1856. New York: Springer, 2004, 165-253

[59] Rockafellar R T. Conjugate duality and optimization. Regional Conference Series in Applied Mathematics, Vol. 16. Philadelphia: SIAM, 1974 
Recent progress in random metric theory and its applications to conditional risk measures

[60] Rosazza Gianin E. Risk measures via $g$-expectations. Insurance Math Econom, 2006, 39: 19-34

[61] Ruszczyński A, Shapiro A. Optimization of convex risk functions. Math Opers Res, 2006, 31: $433-452$

[62] Schachermayer W. A Hilbert space proof of the fundamental theorem of asset pricing in finite discrete time. Insurance Math Econom, 1992, 11: 249-257

[63] Schweizer B, Sklar A. Probabilistic Metric Spaces. New York: Elsevier, 1983; reissued by New York: Dover Publications, 2005

[64] Song Y, Yan J A. The representations of two types of functionals on $L^{\infty}(\Omega, \mathcal{F})$ and $L^{\infty}(\Omega, \mathcal{F}, P)$. Sci China Ser A, 2006, 49: 1376-1382

[65] Song Y, Yan J A. Risk measures with comonotonic subadditivity or convexity and respecting stochastic orders. Insurance Math Econom, 2009, 45: 459-465

[66] Song Y, Yan J A. An overview of representation theorems for static risk measures. Sci China Ser A, 2009, 52: 1412-1422

[67] Vuza D. The Hahn-Banach theorem for modules over ordered rings. Rev Roumaine Math Pures Appl, 1982, 9: 989-995

[68] You Z Y, Guo T X. Pointwise best approximation in the space of strongly measurable functions with applications to best approximation in $L^{p}(\mu, X)$. J Approx Theory, 1994, 78: 314-320

[69] Zowe J. A duality theorem for a convex programming problem in order complete lattices. J Math Anal Appl, 1975, 50: 273-287 